Diana Marcela

Escandón Barbosa*

Andrea

Hurtado Ayala ${ }^{* * *}$

Carlos Alberto

Castillo López

Universidad del Valle, Colombia

Recibido: 13 de febrero de 2013

Concepto de evaluación: 10 de abril de 2013

Aprobado: 7 de mayo de 2013

Artículo de revisión

*Economista, magíster en ciencias de la organización,

candidata a doctora en administración de la Universidad del Valle, docente e

investigadora de la Pontifica Universidad Javeriana. Cali, Colombia.

Correo electrónico: dmescandon@javerianacali.edu.co

**Economista y contadora pública, candidata a magíster en ciencias de la organización de la Universidad del Valle, asistente de docencia de la Universidad del Valle.

Cali, Colombia

Correo electrónico: andrea.hurtado.ayala@ correounivalle.edu.co

***Estudiante de la Maestría en Educación y TIC, apoyo logístico a Educación Continúa y Consultoría de la Pontificia Universidad Javeriana. Cali, Colombia.

Correo electrónico: carlos.castillo@javerianacali.edu.co
Finanzas y Política Económica, ISSN: 2248-6046, Vol. 5, No. 1, enero-junio, 2013, pp. 121-150

\section{Dinámica exportadora de las empresas born global en Colombia}

\section{RESUMEN}

Este artículo pretende analizar las condiciones principales del mercado exportador de las empresas born global en el caso colombiano, teniendo en cuenta los principales factores del entorno que determinan su dinámica exportadora. Se utiliza la base de datos GEM Colombia del año 2011, para analizar 297 empresas born global colombianas, mediante un análisis descriptivo y la estimación de un modelo de conglomerados bietápico. Los resultados muestran que los cambios tecnológicos, las características sectoriales y la intensidad competitiva son factores determinantes de la dinámica exportadora de las empresas born global en Colombia, y se concluye que los empresarios se adaptan al mercado internacional mejorando continuamente sus productos y procesos.

Palabras clave: born global, dinamismo exportador, competencia, tecnología, sectores económicos.

\section{Clasificación JEL: F20, O33, O54}

\section{Export dynamics of born global companies in Colombia}

\section{ABSTRACT}

This article means to analyze the main conditions of the export market for born global companies in Colombia, considering the factors in the context that primarily determine their export dynamics. For that matter we used the GEM Colombia 2011 database to analyze 297 Colombian born global firms through a descriptive analysis and through the estimation of a two-stage cluster model. Results show that technological changes, sectorial characteristics and competitive intensity are factors that determine the export dynamics of born global companies in Colombia. The paper finally concludes that entrepreneurs adapt to the international market by constantly improving their products and processes.

Keywords: born global, export dynamism, competence, technology, economic sectors.

JEL Clasification: F20, O33, O54 


\section{Dinâmica exportadora das empresas born global na Colômbia}

\section{RESUMO}

Este artigo pretende analisar as condições principais do mercado exportador das empresas born global no caso colombiano, considerando os principais fatores do ambiente que determinam sua dinâmica exportadora. Utiliza-se a base de dados GEM Colômbia de 2011 para analisar 297 empresas born global colombianas, por meio de uma análise descritiva e de uma estimativa de um modelo de conglomerados two-stage. Os resultados mostram que as mudanças tecnológicas, as características setoriais e a intensidade competitiva são fatores determinantes da dinâmica exportadora das empresas dessas empresas na Colômbia, e se conclui que os empresários se adaptam ao mercado internacional ao melhorar continuamente seus produtos e processos.

Palavras-chave: born global, dinamismo exportador, competência, tecnologia, setores econômicos. 


\section{INTRODUCCIÓN}

El estudio del dinamismo exportador a nivel global se ha visto reflejado en varias revistas académicas reconocidas a nivel internacional. Estos resultados son diferenciables, según el tipo de empresas (tamaño), factores de carácter interno o externo, ubicación geográfica, entre otros. En general, la mayoría de las teorías tradicionales sobre internacionalización respaldan la existencia de un proceso llevado a cabo por las empresas para entrar a los mercados internacionales, en el cual se asume que las operaciones a gran escala son un requerimiento para las empresas, donde los procesos de internacionalización tardan más tiempo en realizarse. Adicionalmente, estas teorías se enfocan en la existencia de actividades de inversión directa en el exterior para las empresas multinacionales, y unas pocas consideran la internacionalización en pequeñas y medianas empresas nacionales (Oviatt \& McDougall, 1994). Sin embargo, las investigaciones en años recientes giran en torno a nuevas tendencias de internacionalización que han ido apareciendo en el mercado global, lo que ha conducido a generar nuevas propuestas de modelos de internacionalización analizados desde el campo de estudio del entrepreneurship y los negocios internacionales.

Teniendo en cuenta lo anterior, en el campo del entrepreneurship, la creación de empresas se considera una actividad central del empresario (Cuervo, Ribeiro \& Roig, 2007), que puede ser explicada, desde el punto de vista del individuo, con el estudio del ejercicio empresarial como un atributo del ser humano (Kihlstrom \& Laffont, 1979); desde el punto de vista del entorno, a partir del estudio de la dinámica de los mercados (Tushman \& Anderson, 1986), o desde el punto de vista institucional.

La consolidación a nivel mundial de la internacionalización de las nuevas empresas conocidas como born global exige la investigación de este tipo de empresas, las cuales, desde sus inicios, tienen unos objetivos de aprovechamiento de las oportunidades del mercado global. Con base en la revisión de literatura correspondiente, este artículo plantea las condiciones características de las empresas born global y se centra en describir los factores que influyen en el dinamismo exportador de estas empresas en Colombia. Esto se hace considerando: la tecnología, la competencia, y el sector económico (Madsen, 1987), factores del entorno que son esenciales para analizar la dinámica del mercado de estas empresas con base en sus características distintivas (Aaby \& Slater, 1989).

Este trabajo está dividido de la siguiente forma: 1) presentación del marco teórico y la evidencia empírica del tema de investigación, 2) descripción de la metodología utilizada para el análisis de las variables del estudio, 3) resultados del estudio obtenidos por medio de una caracterización del entorno y un modelo de análisis de conglomerados. Finalmente, se presentan las conclusiones y recomendaciones del trabajo.

\section{REVISIÓN DE LA LITERATURA}

En los estudios sobre la internacionalización de empresas es importante reconocer el aporte de cada una de las teorías, que explican cómo las empresas asumen sus procesos de entrada al mercado externo. En este apartado, se hace una breve descripción de las diferentes teorías de internacionalización de empresas, haciendo especial énfasis en la teoría del emprendimiento internacional como nueva perspectiva que da origen al fenómeno de las empresas born global.

\section{Teorías de la internacionalización}

La literatura sobre el estudio de los negocios internacionales inició su construcción enfocándose en el crecimiento de empresas multinacionales (Dunning, 1958; Hymer, 1960). Sin embargo, al final de los años ochenta, la investigación en esta área se focalizó en los mercados de riesgo de capitales y en el emprendedurismo (entrepreneurship) que se observaba especialmente en las empresas orientadas a la tecnología, que a pesar de ser pequeñas compañías se inclinaban a extender sus operaciones hacia otros países industrializados, 
situación que fue determinante en el surgimiento del entrepreneurship internacional en las pymes.

Ohe, Honjo, Oliva y MacMillan (1991) y Rossman (1984) investigaron la existencia de internacionalización independiente del tamaño de la empresa y de los estudios sobre marketing exportador. Ellos siguieron esta línea investigativa caracterizando el proceso de internacionalización a través del cumplimiento de etapas como una explicación a la expansión internacional de las empresas multinacionales (Johanson \& Vahlne, 1977). Este enfoque fue duramente criticado en muchos estudios que han manifestado la existencia de teorías de internacionalización diferentes para empresas de determinado sector, por ejemplo, el caso del sector servicios (Engwall \& Wallenstall, 1988), conformado por empresas que poseen otras características, conocimientos, formas de producción y consumo. Bajo estas críticas, Young (1987) identificó casos en donde la internacionalización se presenta con mayor rapidez, como en las empresas de alta tecnología con altos niveles de investigación, ciclos de productos cortos y concentración del mercado para productos de tecnología.

Teniendo en cuenta el desarrollo de la investigación sobre internacionalización de empresas, se han realizado estudios desde diferentes perspectivas, lo cual ha dado como resultado la aparición de diversas teorías que han buscado explicar este fenómeno. Entre las teorías que han sido utilizadas para explicar el proceso de internacionalización de las empresas pueden encontrarse cuatro enfoques. Primero, se encuentra la perspectiva económica que busca definir cada una de las teorías que intentan explicar las razones que conducen a la expansión internacional de las empresas. Los primeros modelos teóricos bajo esta perspectiva se encuentran basados en la teoría de la firma y la teoría de la organización industrial (Bain, 1959), que plantean la inversión extranjera como producto de las imperfecciones del mercado existentes en mercados monopólicos y oligopólicos.

El segundo enfoque es la perspectiva de procesos, que considera que la internacionalización de una empresa se realiza a través de un proceso donde se acumula conocimiento de los mercados exteriores (Rialp \& Rialp, 2001). Dentro esta perspectiva, se incluye el enfoque de la Escuela de Uppsala, el enfoque de innovación y el modelo de ciclo vida del producto.

El tercer enfoque se centra en la perspectiva estratégica, y explica la internacionalización de empresas a partir de la integración de los recursos de las empresas, a través de la conformación de redes y de la forma como las empresas logran adaptarse a los mercados internacionales. Las teorías que hacen parte de esta perspectiva son la teoría de redes y la teoría de los recursos y capacidades (Mitchell, 1969; Tichy, 1981; Weimann, 1989).

El cuarto enfoque aparece a inicios del siglo $X X I$, como nueva perspectiva de la internacionalización con la aparición del concepto de entrepreneurship internacional, un nuevo campo de estudio que surge de la convergencia entre entrepreneurship (emprendimiento) y negocios internacionales (McDougall \& Oviatt, 1996; Zahra, Ireland \& Hitt, 2000). Bajo este nuevo enfoque, se presenta el fenómeno de las empresas born global, que son empresas internacionales de reciente creación, las cuales se internacionalizan en los primeros años de existencia.

\section{Entrepreneurship internacional}

En general, la mayoría de las teorías tradicionales descritas en los acápites anteriores respaldan la existencia de un proceso llevado a cabo por las empresas para entrar a los mercados internacionales, asumiendo que las operaciones a gran escala son un requerimiento para las empresas multinacionales, donde los procesos de internacionalización tardan más tiempo en realizarse. Adicionalmente, estas teorías se enfocan en la existencia de actividades de inversión directa en el exterior para las empresas multinacionales, y unas pocas consideran la internacionalización en pequeñas y medianas empresas nacionales (Oviatt \& McDougall, 1994). Sin embargo, las investigaciones en años recientes giran en torno a nuevas tendencias de 
internacionalización que han ido apareciendo en el mercado global, lo que ha conducido a generar nuevas propuestas de modelos de internacionalización analizados desde el campo de estudio del entrepreneurship y los negocios internacionales (Jones, Coviello \& Tang, 2011).

Actualmente, es reconocido el uso de conceptos como espíritu empresarial o emprendimiento (entrepreneurship), este último relacionado con empresarios que poseen ciertas habilidades que les permiten obtener resultados exitosos. Shane y Venkataraman (2000) señalan que el entrepreneurship, como campo de estudio, se define por ser un proceso empresarial en el que se identifican y evalúan oportunidades, a partir de lo cual se decide su forma de explotación y las estrategias para llevarlo a cabo, lo cual a menudo conduce a la creación de una nueva actividad económica o una nueva organización.

El entrepreneurship ha favorecido el desarrollo económico al contribuir en la identificación de nuevas oportunidades de negocio, creación de nuevas empresas o mejoramiento de sus procesos, impulso de la innovación y la competencia. Por esto, la literatura económica reciente ha centrado el interés en este tema. Es así como los investigadores no solo estudian a las grandes compañías multinacionales, sino que han incluido también a las firmas emprendedoras como unidad de análisis, teniendo en cuenta, el incremento del comercio internacional y la acelerada internacionalización de pequeñas y nuevas empresas, producido por la liberalización de los mercados y el desarrollo de las tecnologías de la información. Esto ha llevado al surgimiento del concepto international entrepreneurship, definido como una combinación de conducta innovadora, proactiva y arriesgada que cruza las fronteras nacionales e intenta crear valor en las organizaciones (McDougall \& Oviatt, 2000). Posteriormente, Oviatt y McDougall (2005) complementan esta definición agregando que esta búsqueda de oportunidades en el mercado global también permite crear nuevos productos o servicios. International entrepreneurship o emprendimiento internacional se describe como un proceso organizacional de largo alcance, que tiene como fin generar valor con el aprovechamiento de las oportunidades en el mercado internacional (Dimitratos \& Plakoyiannaki, 2003). Autores como Lumpkin y Dess (1996) asocian el entrepreneurship con la decisión del emprendedor sobre la entrada a un mercado, mientras que autores más recientes como Perks y Hughes (2008), Zahra y George (2002), Dimitratos y Jones (2005) señalan que las definiciones iniciales del international entrepreneurship se concentran en las nuevas pymes que realizan rápidos procesos de internacionalización. Por lo tanto, el fenómeno de las nuevas empresas internacionales ha sido uno de los temas centrales en el campo de investigación del international entrepreneurship, a partir del cual surge el concepto de las empresas born global, que nacen para los mercados internacionales, cuyo estudio inicia en los años noventa, en donde se conjugan los aportes del entrepreneurship y del comercio internacional.

Estos emprendimientos globales pueden llegar a tener diversas implicaciones de orden nacional e internacional. Rennie (1993), Madsen y Servais (1997) señalan que las pequeñas y medianas empresas juegan un importante papel en el crecimiento económico de un país, pero, a su vez, al ser empresas internacionales presentan ciertas características que limitan su capacidad exportadora, como el acceso a la información, la tecnología, y en algunos casos son poco innovadoras, y presentan dificultades para el acceso a la financiación. Adicionalmente, los gerentes deben enfrentar diferentes circunstancias asociadas a estas características, sobre todo aquellas relacionadas con el enfrentamiento a la competencia, porque, según lo sugerido por Moen (1999), gran parte de estas empresas presentan rezagos tecnológicos y muchas veces es difícil que implementen nuevas herramientas tecnológicas (Knigth, 1997). Por tanto, los gerentes de las empresas born global tienen entre sus principales retos, ser mucho más arriesgados en asumir los cambios tecnológicos y aprender a realizar una continua revisión de la competencia internacional, para intentar desarrollar nuevas ventajas competitivas que aseguren su éxito exportador, 
seleccionando segmentos del mercado donde puedan enfocar sus recursos.

Rasmussan et al. (2001) Rasmussan y Madsen (2002) concluyeron en sus estudios, que la internacionalización de las empresas no es el principal objetivo del entrepreneur en el proceso de creación de empresas, y afirman que las born global son creadas específicamente por la necesidad de independencia por parte del empresario, acompañada del surgimiento de nuevas empresas que cuentan con el apoyo de una red empresarial. Dentro de este estudio también se encuentra que algunas empresas de este tipo fueron creadas sin necesidad del apoyo de una red u organización en donde necesariamente estuviera incluido el empresario, por lo tanto, se concluye que la creación de una nueva empresa internacionalizada puede surgir de nuevas ideas y oportunidades, acompañada de la experiencia del empresario.

\section{El concepto de nueva empresa internacional o born global}

Los cambios presentados en la economía mundial durante los últimos años han llevado al surgimiento de nuevas teorías que intentan complementar los modelos tradicionales sobre los procesos de internacionalización de las empresas. Entre estas nuevas teorías, la más relevante es la relacionada con el fenómeno de las born global o international new ventures, enfocada en las pymes que nacen internacionalizadas.

Las pymes, que desde el inicio de sus actividades hacen presencia en el mercado internacional, han sido denominadas como born globals. Los estudios de McKinsey y Co. (1993) y Rennie (1993) introducen el concepto de empresa born global; sobre esta base, Oviatt y McDougall (1994) realizan una de las definiciones más aceptadas de las nuevas empresas internacionales, pues las consideran como una organización empresarial que desde sus inicios busca la obtención de ventajas competitivas usando recursos de origen internacional y vendiendo productos a varios países. Otros trabajos como los de Knight y Cavusgil (1996) o Madsen y
Servais (1997) complementan esta definición. En primer lugar, Knight y Cavusgil (1996) pretenden justificar la existencia de estas empresas analizando el comportamiento de algunas pequeñas empresas de tipo tecnológico que operan en mercados internacionales desde los primeros años de su fundación y que son dirigidas por empresarios con una amplia visión hacia la expansión de fronteras. En segundo lugar, Madsen y Servais (1997) buscan dar más precisión a la definición de Oviatt y McDougall (1994), concluyendo que las empresas born global son creadas con un alcance comercial ilimitado, donde su expansión es de ámbito internacional (Luostarinen \& Gabrielsson, 2004). Por lo tanto, la velocidad y el crecimiento de la internacionalización temprana reflejan la existencia de una oportunidad que está siendo explotada (Autio, Sapienza \& Almeida, 2000; Oviatt y McDougall, 2005).

Dentro de las definiciones de empresa born global, no existe consenso en el número de años que transcurre desdeque la empresa se crea hasta que se internacionaliza (Acedo \& Jones, 2007), cifra que oscila desde los dos años después del comienzo de su actividad (McKinsey \& Co., 1993; Rennie, 1993; Knight \& Cavusgil, 1996), los tres años (Madsen \& Servais, 1997; Iborra, Menguzzato \& Ripollés, 1998; Knight, Madsen \& Servais, 2004; Servais, Zucchella \& Palamara, 2006; Zuchella, Palamara \& Denicolani, 2007), los seis años (Shrader, Oviatt \& McDougall, 2000; Zahra, Ireland \& Hitt, 2000), los siete años (Jolly, Alahunta \& Jeannet, 1992), los ocho años (McDougall \& Oviatt, 1996) y hasta los diez años (Milanov \& Fernhaber, 2009). Otro de los criterios para identificar a estas empresas es el porcentaje de ventas en el exterior, en el que muchos autores coinciden en un mínimo del $25 \%$ de la facturación procedente de mercados exteriores (Kuivalainen, Sundqvist \& Servais, 2007; Milanov \& Fernhaber, 2009). Iborra et al. (1998) y Ripollés et al. (2002) también destacan que las empresas born global poseen un grado de internacionalización elevado, y comprueban que son empresas que consideran la internacionalización no como una alternativa, sino como respuesta a su actitud proactiva. 
Rialp y Rialp (2001) sintetizan las principales características de las nuevas empresas internacionales en nueve factores: una concepción global desde la creación de la empresa, directivos con alta experiencia internacional, compromiso de gestión, utilización de redes, acceso a recursos intangibles basados en el conocimiento, creación de valor, centralización en nichos de mercado, alta orientación hacia el cliente y adaptabilidad a los cambios del entorno.

Para efectos de este artículo, y teniendo en cuenta las diferentes definiciones de born global presentadas, se plantea el siguiente concepto unificado de estas empresas: como aquellas empresas nuevas o recientemente creadas que alcanzan su éxito exportador paralelamente con su proceso de creación y consolidación, y para ello asumen una actitud proactiva frente a las oportunidades que presenta el mercado.

Por otra parte, además del interés de los investigadores por definir a las empresas born global, también se han preocupado por conocer cuáles son los factores que han favorecido el cambio del comportamiento particular de estas empresas. Estos factores son de origen interno y externo (Knight \& Cavusgil, 1996; 2004; Madsen \& Servais, 1997; Knight, 2000), y están catalogados en cuatro grupos:

1. El entorno internacional (Oviatt \& McDougall, 2005), representado por el cambio económico, tecnológico y social.

2. El sector al que pertenece la empresa. En sus inicios la presencia de empresas born global se relacionó con sectores intensivos en tecnología (Bell, 1995; Oviatt \& McDougall, 1995; Knight \& Cavusgil, 1996; Madsen \& Servais, 1997; Autio \& Sapienza, 2000; Autio et al., 2000), pero recientes investigaciones han encontrado que este tipo de empresas pueden encontrarse en diferentes industrias (Madsen \& Servais, 1997; McAuley, 1999; Knight, 2000; Rialp et al., 2002, 2005; Zahra, 2005; Blomqvist et al., 2008).
3. La estrategia de la empresa que asocia factores como la especialización en nichos de mercado (Galván, 2003), la inversión en I+D y penetración simultánea en diversos mercados (Oviatt \& McDougall, 1995).

4. La actitud de los directivos (Madsen \& Servais, 1997; Zahra \& George, 2002; Oviatt \& McDougall, 2005; Zucchella et al., 2007; Nordman \& Melen, 2008) caracterizada por la experiencia internacional, su conocimiento de idiomas extranjeros, su nivel educativo, un carácter proactivo, la percepción de oportunidades de crecimiento en el exterior, entre otras características.

Las empresas born global presentan algunas ventajas sobre las empresas con un proceso de internacionalización tradicional (Rennie, 1993), correspondientes con las relaciones entre empresa y mercado, tales como:

1. El cambio en las preferencias del mercado: donde los consumidores demandan productos mucho más especializados que favorecen a las empresas born global, que por ser pymes poseen una mayor flexibilidad para adaptarse a estos cambios de necesidades.

2. Cambios en las condiciones competitivas: generalmente, las empresas más pequeñas suelen ser más adaptativas y controlar mejor sus costos.

3. Conocimiento del cliente: estas empresas comprenden que la satisfacción del cliente es una de sus principales herramientas para abrir mercados.

Por otra parte, Jolly et al. (1992) señalan que existe una serie de desventajas relacionadas específicamente con el tamaño de estas empresas. Por lo general, estas empresas son pequeñas y medianas, con capacidades limitadas para enfrentarse a otros mercados, porque su capacidad instalada no supera los estándares necesarios para ello. Entre sus principales desventajas están: 
1. La dependencia en un solo producto es un limitante para expandir mercados, dado que estas empresas deben centrarse en cubrir los mercados donde su producto sea líder.

2. Las distancias físicas: la elección de un mercado depende de la distancia geográfica, por esto, muchas veces las empresas born global deben valerse de redes y contactos internacionales para conducir sus ventas, buscando socios complementarios.

En general, las empresas born global requieren tener cercanía con sus clientes si su objetivo es generar valor y ser exitosas, por lo tanto, si se centran en satisfacer las necesidades de un determinado grupo de clientes, dirigen la creación de un mercado exclusivo.

Cabe anotar que, con la existencia de nuevos enfoques como la teoría de los recursos y capacidades y el enfoque born global, se logra recuperar el carácter dinámico del proceso de internacionalización que carece de perspectiva económica e incluso de perspectiva de procesos. La nueva literatura tiende a generar modelos que permitan integrar aspectos importantes de cada una de las perspectivas para alcanzar un modelo mucho más realista y cercano al entorno actual de las empresas (Cuervo et al., 2007).

\section{El dinamismo del mercado exportador de las empresas born global}

Teniendo en cuenta las características particulares que poseen las empresas born global, este artículo se centra en analizar la dinámica del entorno, como aspecto importante para que una empresa nueva tome la decisión de entrar en el mercado (Bartlett \& Ghoshal, 1991). Shoham (1999) menciona la complejidad de un entorno internacional y la necesidad de evaluar las capacidades de las empresas, los cambios en las condiciones del negocio, el entorno competitivo, una tarea bastante complicada en entornos donde interactúa el mercado nacional e internacional. Para Shoham (1999), el entorno empresarial está compuesto por un gran número de factores sociales, políticos, y culturales, y las condiciones dentro y fuera del mercado pueden influir en estos factores, las cuales se convierten en parte del correspondiente entorno (Bartlett \& Ghoshal, 1991).

Factores como la intensidad competitiva y el dinamismo del mercado se consideran significativamente influyentes en las iniciativas estratégicas de las empresas internacionales (Jap, 1999; Jaworski \& Kohli, 1993). De esta manera, la intensidad o rivalidad competitiva es el grado de competencia que enfrenta una empresa en el mercado (Kohli et al., 1993). El nivel de intensidad competitiva está relacionado con las actividades competitivas de las empresas en el mercado, incluido el precio, la promoción, entre otros. Al entrar en un mercado extranjero altamente competitivo, las empresas se enfrentan al reto de adquirir una cuota de mercado y el establecimiento de una posición competitiva en el mercado a través de filiales. Las empresas deben proporcionar a los clientes locales productos de calidad única, lo cual es clave para la construcción de ventaja competitiva en el mercado. La transferencia tecnológica es una de las formas para mejorar la calidad de los productos, pues reduce los costos de producción y los precios a competir.

Jaworsky y Kohli (1993) definen el dinamismo del mercado como la "existencia de cambios en un mercado, derivado de la modificación en la composición de clientes y competidores, o de la variación de las preferencias de los primeros". Por tanto, ante la existencia de un mercado internacional dinámico, la empresa debe analizar constantemente las necesidades de los clientes actuales y la exploración de las preferencias de los clientes potenciales, con lo cual se debe conformar un portafolio de productos armónico con estos cambios. En este sentido, las empresas en un entorno dinámico de tipo internacional tienden a promover la existencia de un alto grado de orientación a esos mercados internacionales.

Una de las variables que se considera como determinante de las exportaciones es la tasa de 
cambio real, teniendo en cuenta que los procesos de devaluación o revaluación influyen directamente sobre el crecimiento de las exportaciones. Sin embargo, el análisis comparativo entre el comportamiento de la tasa de cambio y las exportaciones generalmente se realiza por medio del estudio de series de tiempo, y para este estudio se utilizará una base de datos de corte trasversal, situación metodológica por la que se excluye esta variable del estudio.

En general, todos los cambios en la dinámica mundial han generado que la estructura empresarial tienda a seguir estas exigencias actuales; una evidencia de esto es la formación de pequeñas empresas que se aventuran a competir directamente en mercados internacionales. Es decir, las empresas born global que logran analizar un entorno, en el cual, a pesar de ser altamente cambiante, logran orientarse al mercado internacional por medio de la recopilación de información de datos de diferente tipo (Andersen \& Buvik 2002) y detectar posibles oportunidades que las reflejan en la internacionalización temprana o la expansión a nuevos mercados (Rialp, 1999).

Jaworsky y Kohli (1993) sugieren que el entorno de una organización puede influir en su nivel de internacionalización; es decir, que las organizaciones en entornos más competitivos pueden llegar a estar más internacionalizadas e incluso argumentan que la relación entre las exportaciones y el rendimiento depende de las características del entorno de una organización (Kohli \& Jaworski, 1990).

En empresas que hacen parte del entorno internacional, los factores ambientales tienen una importante influencia sobre la estrategia y el éxito de estas organizaciones que operan en los mercados extranjeros (Carpano, Chrisman \& Roth, 1994). En el caso de las empresas born global, los entornos específicos de cada país construyen la naturaleza e intensidad de la competencia e influyen en los mecanismos para hacer transacciones entre organizaciones, y afectan la dinámica de entrada y salida en las industrias. Por lo tanto, este artículo se centra en caracterizar los factores ambientales que influyen en las iniciativas de una internacionalización temprana en las empresas colombianas, donde aspectos sectoriales, ubicación geográfica y cambios tecnológicos conllevan a que el mercado fluctúe continuamente, afectando los resultados exportadores de las empresas denominadas born global (Luo \& Park, 2001; Zou, Taylor \& Osland, 1998; Katsikeas, Piercy \& loannidis, 1996; Sousa, Martínez-López \& Coelho, 2008).

Diversos estudios sobre el fenómeno de las empresas born global muestran algunos factores característicos del entorno de estas empresas, que influyen en su dinámica exportadora. Bloodgood, Sapienza y Almeida (1996) examinan las características estratégicas y los resultados de la internacionalización de las nuevas empresas, ellos encontraron que la internacionalización temprana depende del tipo de industria y las condiciones de los recursos de la empresa. Knight y Cavusgil (1996) proponen que los factores que promueven los esfuerzos internacionales de las empresas born global son los nichos del mercado, los avances en las tecnologías y las redes globales. Madsen y Servais (1997) señalan que el desarrollo de la empresa born global es afectado por las características del entorno, de la organización y del fundador. Por otra parte, Madsen, Rasmussen y Servais (2000) estudian la estructura y conducta de las empresas born global en Dinamarca, ellos resaltan que poseen métodos de producción especiales, un alcance geográfico y un comportamiento proactivo. Moen (2002) manifiesta que las empresas born global se caracterizan por una orientación internacional y estrategia de la exportación similar a las empresas tradicionales; asimismo, la dinámica exportadora de la empresa parece ser determinada desde su fundación, y las condiciones del mercado son factores importantes que explican por qué algunas empresas son born global. Por último, Monreal (2009) determina que el comportamiento exportador de las empresas born global se basan en el entorno en el que la empresa se mueve, el cual inicia desde los facto- 
res del mercado local y las características de los mercados destino como el tipo de industria, el contenido tecnológico, la rivalidad competitiva, la situación económica del país y las condiciones de demanda interna.

También existe evidencia empírica de la utilización de los modelos de conglomerados como metodología para el análisis característico de las empresas. Entre los estudios más recientes se encuentra Díaz, Moniche y Morillas (2005), quienes proponen un análisis de conglomerados en dos etapas para clasificar a los sectores productivos en España, con base en variables que describen el comportamiento en la estructura organizacional de la tabla input-output española de 1995, así como en información externa a esta tabla, como, por ejemplo, el nivel tecnológico, la innovación, entre otros. Con esta metodología, los autores buscan revisar la pertenencia de un sector a los diversos grupos conformados para las semejanzas entre variables. Flor y Oltra (2009) examinan las estrategias seguidas por las empresas en los mercados exteriores, para identificar dichas estrategias recurren al análisis de conglomerados jerárquicos, tomando variables como la dispersión geográfica; la adaptación del marketing mix; los métodos competitivos, como dimensiones seleccionadas para representar las estrategias. Fernández, Peña-García y Hernández (2008) buscan los factores determinantes del éxito exportador determinados a partir de las estrategias desarrolladas en los mercados exteriores que se obtienen por medio de un análisis de conglomerados jerárquico. González, Peña y Vendrell (2012) prueban el grado en que las diferencias en las capacidades organizacionales y la creación de nuevas empresas difieren entre regiones, estas diferencias están asociadas con el nivel competitividad regional y para ello utilizan el análisis de conglomerados jerárquicos para clasificar a las regiones españolas a lo largo de las dimensiones innovación y capacidad empresarial.

\section{METODOLOGÍA}

Los resultados de este estudio se obtienen a través de un método de análisis cuantitativo, que parte de la base de datos GEM, para estimar el método de análisis multivariante conocido como análisis de conglomerados o análisis clúster, que permite clasificar aquellas características que determinan la dinámica exportadora de las empresas born global. Por lo tanto, en este apartado se encontrará una descripción de la muestra utilizada y la explicación del modelo estimado, al igual que la descripción de las variables utilizadas.

\section{Muestra}

Los resultados de este estudio se obtienen a través del análisis cuantitativo de una serie de variables extraídas de la base de datos de GEM (Global Entrepreneurship Monitor) Colombia 2011. En Colombia, para el año 2011, al igual que en los años anteriores, la encuesta fue aplicada por el Centro Nacional de Consultoría (CNC). En ella se formularon telefónicamente un poco más de 10.000 cuestionarios en los principales centros urbanos del país (Pereira, Bucaramanga, Manizales, Barranquilla, Cartagena, Santa Marta, Sincelejo, Bogotá, Medellín y Cali), y en otras ciudades del territorio nacional y en otros 23 municipios de áreas rurales seleccionadas aleatoriamente con población inferior a 10.000 habitantes (Escandón y Hurtado, 2012).

Dentro de los criterios que son tenidos en cuenta para obtener los datos relacionados con empresas born global, se utilizan tres puntos importantes para seleccionar este tipo de empresas en la base de datos GEM.

1. El año de fundación de las empresas no debe superar los 7 años de constitución. Para este caso, el año de creación de las empresas debe ser posterior al 2003.

2. Las empresas deben tener actividad exportadora, con por lo menos, $25 \%$ de ventas anuales en el exterior. 
3. No incluir empresas subsidiarias o filiares. Para el caso colombiano este criterio no arrojó resultados.

A partir del cumplimiento de estas condiciones, se sustrae una muestra de 297 empresas colombianas.

\section{Modelo}

El análisis clúster o de conglomerados es un conjunto de técnicas multivariantes, cuyo propósito es agrupar objetos según las características que poseen. Esta técnica clasifica objetos (es decir, encuestados, productos, empresas, entre otros), de tal forma que cada objeto se parece a los que hay en el conglomerado, según cierto criterio de selección predeterminado (Hair, Anderson, Tatham \& Black, 1999). El objetivo principal del análisis clúster es definir la estructura de datos colocando las observaciones más parecidas en grupos.

Las variables seleccionadas para el estudio caracterizan los objetos que se están agrupando; es decir, la dinámica exportadora de las empresas born global colombianas, a partir de la evidencia empírica que sustenta la utilización de estas variables (Luo \& Park, 2001; Zou, et al., 1998; Katsikeas et al., 1996; Sousa et al., 2008). Las variables utilizadas para el modelo son:

1. Nivel tecnológico (medido por la dificultad para predecir la tecnología en los últimos años y el nivel de cambios tecnológicos rompedores).

2. Características sectoriales (medida por la ciudad colombiana donde la empresa está localizada y el sector de actividad económica).

3. Intensidad competitiva (tamaño del mercado, actividad competitiva, cantidad de competidores, tamaño de los competidores).

El análisis de conglomerados bietápicos tiene el propósito de obtener las variables que son más determinantes en la caracterización del perfil de la dinámica exportadora de las empresas born global en Colombia, proceso que realiza por medio de un modelo de agrupamiento que permite el análisis de las variables para establecer su contribución en la caracterización de los distintos grupos. El análisis bietápico toma como métrica de similitud la distancia euclídea (en línea recta) entre cada par de observaciones, cuya fórmula de cálculo es la siguiente:

$$
d_{i j}=\sqrt{\sum_{k=1}^{p}\left(X_{i k}-X_{j k}\right)^{2}}
$$

Donde $d_{i j}$ es el valor de la distancia entre las unidades de análisis $i$ y $j ; X_{i k^{\prime}}$ y $X_{j k}$ son los valores de la variable $k$ para las unidades $i$ y $j$, respectivamente, mientras $p$ es el número de las variables cuyos valores se busca comparar (Escandón \& Hurtado, 2012).

La distancia euclídea clasifica los individuos según su similaridad entre sí. Para medir qué tan similares (o disimilares) son los individuos se toma este indicador basado en la distancia, que considera a los individuos como vectores en el espacio de las variables. La distancia euclídea es el método más utilizado para estos casos, dado que su definición coincide con el concepto más común de distancia (Hair et al., 1999).

El cálculo de esta medida de distancia requiere que las variables sean previamente estandarizadas con el fin de que la escala de medición brinde un valor correcto de las distancias. Los valores de distancia constituyen la matriz de distancia, sobre la cual el programa aplica diferentes algoritmos matemáticos que conducen a la conformación de grupos conformados por aquellos individuos que poseen la mayor similitud (Hair et al., 1999).

En este estudio, la técnica de análisis multivariante de conglomerados bietápico o clúster en dos etapas se realiza por medio del software estadístico SPSS, versión 19, que, por medio de los procedimientos ya descritos, da como resultado un número óptimo de conglomerados que clasifican los 
datos. Para esto se siguió el criterio de información bayesiano de Schwarz, que desarrolla una medida consistente basada en el logaritmo de la función de verosimilitud, el número de parámetros independientes a ser estimados y el tamaño muestral (Schwarz, 1978), incluyendo en su cálculo variables continuas, previamente tipificadas. Esta técnica permitió definir 2 grupos empresariales con características específicas a nivel tecnológico, sectorial y competitivo, según la dinámica del mercado.

\section{RESULTADOS}

Los resultados examinados se integran en dos grandes grupos: el primero es la caracterización del mercado exportador de las empresas, según la clasificación de las diferentes actividades económicas versus sus características tecnológicas, los resultados exportadores con su consecuente localización geográfica y la intensidad competitiva y sus respectivos resultados exportadores. Y el segundo grupo es un análisis de conglomerados que muestra la influencia de estas variables del mercado sobre los resultados exportadores.

\section{Características del mercado}

\section{Distribución geográfica las empresas born global en Colombia}

Dentro del primer grupo de análisis, se relaciona el código CIIU (Clasificación Industrial Internacional Uniforme) con su respectiva descripción (ver anexo 1), lo que permite analizar la distribución geográfica de las born global en los distintos sectores, su tamaño de mercado, sus competidores, su impacto tecnológico y sus resultados exportadores.

El análisis de la distribución geográfica por código CIIU de las empresas born global en Colombia (gráfica 1) permite concluir que el $41 \%$ de estas empresas están ubicadas en Bogotá, el $27 \%$ en Medellín, el $21 \%$ en Boyacá, el $10 \%$ en Cali y el $1 \%$ en Pereira. Por otra parte, la mayoría se concentra en los sectores de fabricación de sustancias y productos químicos (54), fabricación de productos de caucho y de plástico (51), elaboración de productos alimenticios y bebidas (44) y fabricación de productos textiles (28). Estos datos coinciden con la información presentada por Confecámaras (2012), donde de un total de 1.238 .337 sociedades, solo el 0,8\% de las registradas a mayo del 2012 afirman haber exportado en el último año y ubicadas mayoritariamente $(57,5 \%)$ en la región Centro (Bogotá, Cundinamarca, Huila y Tolima), con 5710 compañías, seguidas de Antioquia-Eje Cafetero con 1827 compañías exportadoras (18,4\%). Posteriormente se ubica el Pacífico (Valle del Cauca y Cauca), con 904 compañías (9,1\%), el Caribe con 854 (8,6\%), Oriente (Boyacá y Santander, entre otros), con 616 $(6,2 \%) y$, en último lugar, la Amazonia y Orinoquia, con 20 compañías $(0,2 \%)$. De igual forma, se registran que la mayoría de las empresas exportadores son pymes $(51,1 \%)$, las cuales producen el $82,2 \%$ de las exportaciones del país, con lo cual se ratifica el gran potencial y dinamismo de este tipo de empresas, asociado a una estrategia de desarrollo de productos o procesos innovadores que generan valor y que se ajustan a los mercados, a sus consumidores y a sus culturas.

Es importante resaltar que la dinámica exportadora de las empresas born global está acorde con la fortaleza económica gestada en Colombia en los últimos años y que logra consolidarse en el año 2011 por medio del restablecimiento de la confianza y de los acuerdos comerciales con los países vecinos como Ecuador y Venezuela. Esto sumado al progreso y puesta en marcha de relaciones multilaterales de Colombia, dentro de las que se destacan: 7 tratados de libre comercio con países pertenecientes a la Comunidad Andina, Mercosur, México, Chile, El Salvador, Honduras, Guatemala, Suiza, Canadá y con amplias expectativas con la firma del TLC con Estados Unidos en el 2012 (Ministerio de Comercio, Industria y Turismo, 2012).

Según ANIF (2012), los principales destinos de exportación de las empresas colombianas durante el primer semestre del 2012 son Ecuador, Estados Unidos, Perú, Bolivia, Chile, Venezuela y algunos países de la Unión Europea. 


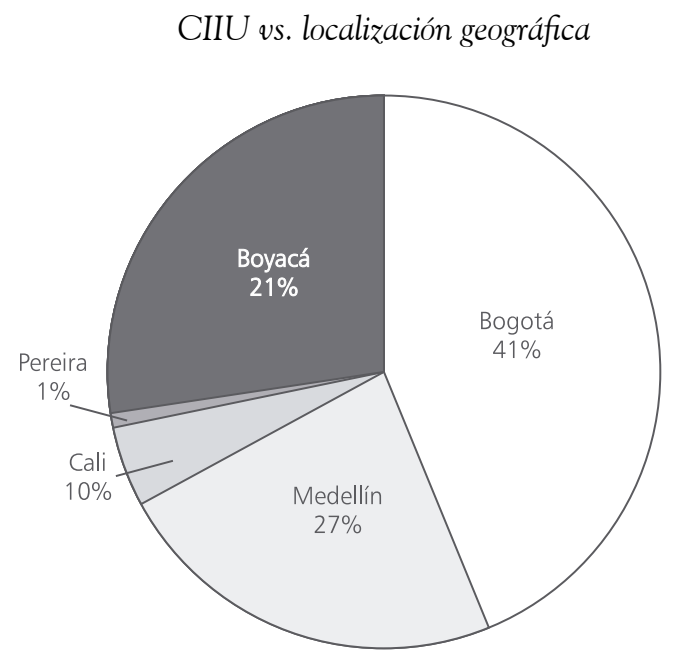

Fuente: Elaboración de los autores.

Gráfica 2 .

Destinos de las exportaciones colombianas

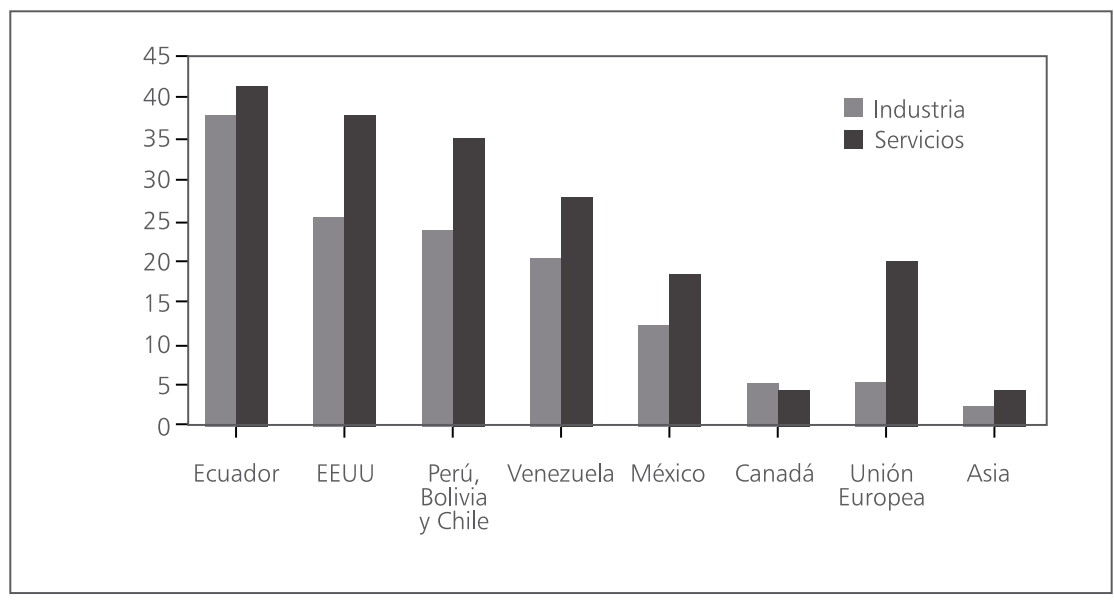

Fuente: Adaptado de ANIF (2012).

También se está logrando abrir mercados no solo en América Latina, sino en otros países con gran potencial para las exportaciones como Canadá, México, Alemania, España, entre otros (gráfica 2).

Algunas características que presentan los mercados potenciales, y que los hacen atractivos para destinar las exportaciones por parte de las empresas colombianas, son (ANIF, 2012):
Estados Unidos por ser uno de los países más grande del mundo, es uno de los importadores más importantes, tiene más de 313 millones de habitantes y cuenta con la mayor diversidad cultural y étnica por la migración de personas de diferentes países.

En cuanto a competidores del mercado, Canadá está posicionado en el puesto 12 en la lista de los mayores importadores a nivel mundial, 
siendo el mayor importador Estados Unidos con un $50 \%$ de participación. Para este país, los productos más apetecidos son del sector manufacturero y, especialmente, los empaques, envases y autopartes.

México es un mercado de gran tamaño, donde se presentan fenómenos como el ajuste demográfico y la migración a otras ciudades, fidelidad a marcas de bajo precio y una preferencia de compras en las grandes superficies. Adicionalmente, Proexport resalta que el $95 \%$ de los productos colombianos (sector manufacturero) gozan de entrada sin aranceles a México, gracias al acuerdo a libre comercio vigente, mientras que otros países deben pagar un arancel del 15\%. Dentro de los productos con mayor potencial se encuentran las confecciones, alimentos, ferreteros, plásticos para el hogar, entre otros.

\section{Impacto tecnológico de las empresas born global en Colombia}

En lo referente al impacto tecnológico en los distintos sectores (CIIU), la gráfica 3 describe la percepción de las empresas clasificadas en los distintos CIIU, sobre la predicción de la tecnología reinante (para los próximos 2-3 años) y el hecho de que en los últimos años se hayan dado cambios tecnológicos rompedores.

En lo referente a los CIIU (fabricación de productos textiles -17-, elaboración de productos alimenticios y bebidas - 15-, fabricación de productos de caucho y de plástico - 25- y fabricación de sustancias y productos químicos -24-) que contienen el mayor número de empresas born global, se resalta que la mayoría (51\%) logra reconocer que es muy difícil predecir la tecnología y que en años recientes se han generado grandes cambios tecnológicos y, por ende, es muy difícil predecir la tecnología reinante para los próximos 2 o 3 años. Es decir, los empresarios son conscientes que la única forma de mantenerse en el mercado internacional es por medio del mejoramiento de sus procesos o productos como una forma de mejorar sus niveles de especialización y su adaptación a las necesidades del cliente. Esto sumado a que la inclusión de nueva tecnología de forma constante puede estar planteándose como una forma de incrementar la rivalidad o establecer altas barreras de entrada al sector (Shrader et al., 2000) y donde cada sector económico se caracteriza por exigir sus propios niveles tecnológicos para

CIIU vs. en años recientes se han producido cambios tecnológicos (rompedores)

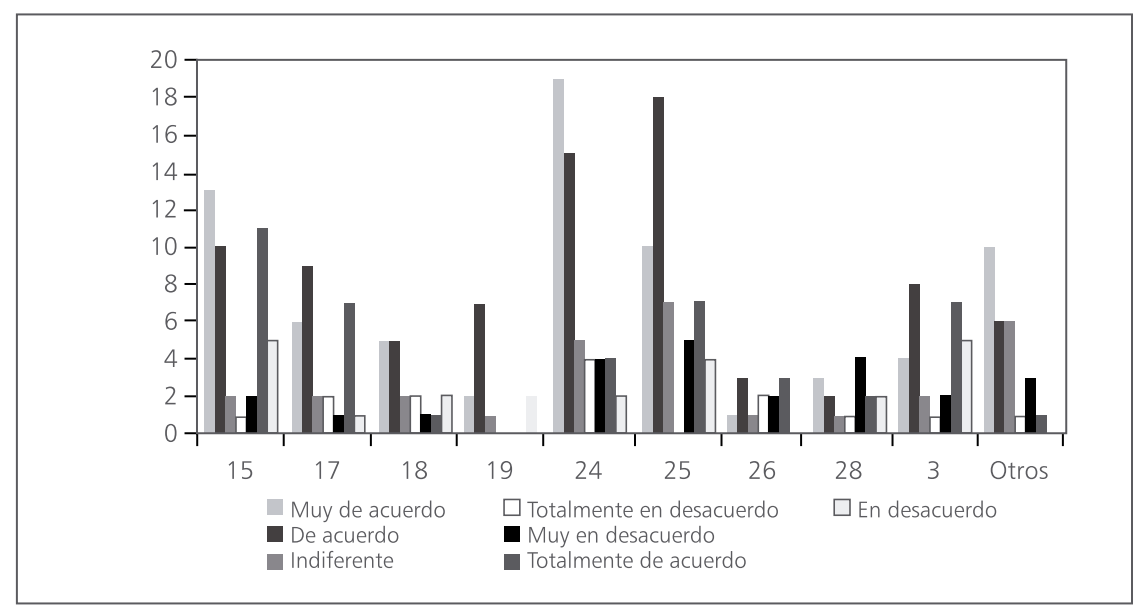

Fuente: Elaboración de los autores. 
establecerlos como requerimiento para ingresar al sector y a la otros mercados por fuera del país de origen.

\section{Intensidad competitiva de las empresas born global}

Por otra parte, respecto a la valoración de los competidores en el mercado, en la gráfica 4 se describe la fuerte tendencia de las empresas en reconocer que existen muchos competidores en el mercado yque los competidores son empresas grandes y fuertes. En resumen, el $86 \%$ de las empresas estiman que existen muchos competidores en el mercado y el $85 \%$ de las empresas valoran a sus competidores como empresas grandes y fuertes.

\section{Actividad exportadora de las empresas born global en Colombia}

En cuanto a la evaluación de las empresas acerca de la actividad exportadora se analiza que de las empresas pertenecientes al CIIU 1 (agricultura, ganadería, caza y actividades de servicios conexas) en promedio el $58 \%$ califica sus exportaciones como muy exitosas; en el CIIU 2 (silvicultura, extracción de madera y actividades de servicios conexas), el $47 \%$ se consideran exitosas y también lo consideran el $51 \%$ de las empresas pertenecientes al CIIU 3 (otros).

Adicionalmente, dentro de los códigos CIIU que agrupan el mayor número de empresas de tipo born global, tienen una mejor percepción de éxito de sus exportaciones los siguientes:

- CIIU 15 (elaboración de productos alimenticios y bebidas), el $87 \%$ consideran las exportaciones como muy exitosas y solo el $2 \%$ consideran que no han sido exitosas.

- CIIU 24 (fabricación de sustancias y productos químicos), el $76 \%$ consideran las exportaciones como muy exitosas y el $8 \%$ consideran que no han sido exitosas.

- CIIU 25 (fabricación de productos de caucho y plástico), el $77 \%$ consideran las exportaciones como muy exitosas y el $11 \%$ consideran que no han sido exitosas.

En tercer lugar, en el anexo 2 se describe el incremento de las exportaciones en los últimos 3 años de las empresas clasificadas en los distintos CIIU, donde el $77 \%$ de las empresas incrementaron las exportaciones, mientras que el $23 \%$ restante no

CIIU vs. actividad competitiva agresiva en el mercado

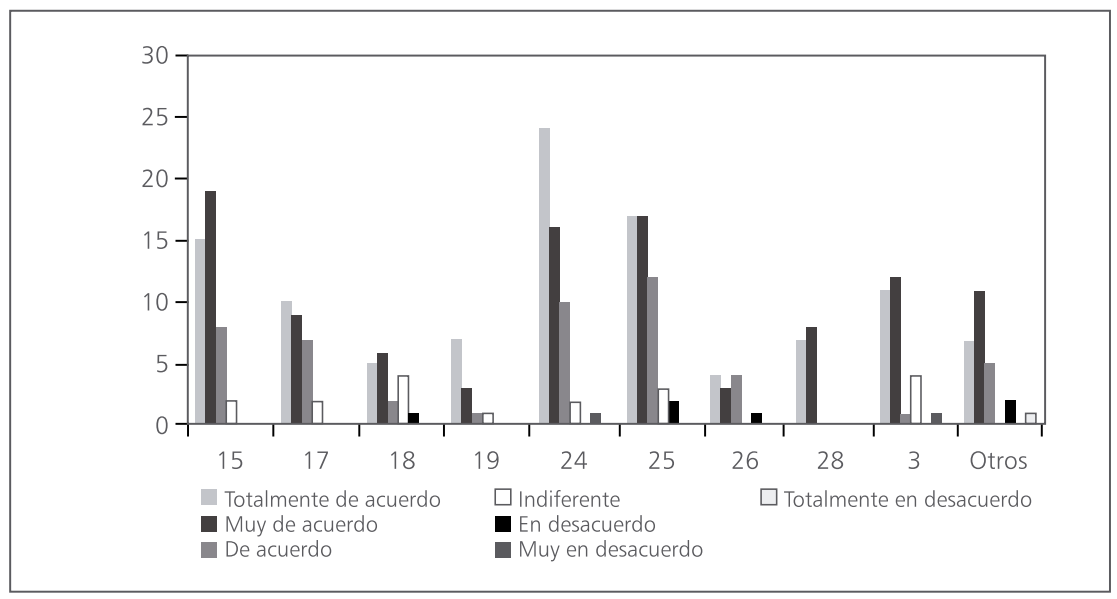

Fuente: Elaboración de los autores. 
lo hicieron. Adicionalmente, el $81 \%$ de empresas que clasificaron las exportaciones con una valoración de muy exitosas fueron aquellas que incrementaron las exportaciones del $10 \%$ al $20 \%$ o más del $20 \%$. Este dinamismo exportador se debe en parte a que en los últimos años el entorno del país ha cambiado significativamente en aspectos como: a) el mejoramiento considerablemente de la situación de seguridad y orden público; (b) aumento en el clima de negocios y el atractivo para la inversión local y extranjera; (c) aumento de las coberturas en educación; (d) se ha avanzado en la agenda de política internacional; (e) avance significativo en el uso y difusión de las tecnologías de la información y comunicaciones, y (f) desarrollo del sistema financiero.

Finalmente, para el análisis del dinamismo exportador, se ratifica que las empresas describen una tendencia muy activa en los mercados internacionales (percepción del tamaño del mercado y actividad competitiva) desde una edad temprana (menos de 4 años de edad) y donde la mayoría de estas empresas hacen de las exportaciones el primer y principal modo de entrada al mercado exterior.

El $80,1 \%$ de las empresas ubicadas en Bogotá, Medellín, Boyacá, Cali y Pereira aumentaron la actividad exportadora en los últimos 3 años más de un $11 \%$. De ese porcentaje, el $71,2 \%$ aumentaron más del $20 \%$ su actividad exportadora en los últimos 3 años (ver anexo 3). Finalmente el mayor número de empresas que aumentaron las exportaciones más del $11 \%$ en los últimos 3 años se encuentran ubicadas en Bogotá (97 empresas) y Medellín (69 empresas).

El análisis de estos resultados permite inferir que la influencia de la ubicación geográfica está directamente relacionada con el resultado de las exportaciones en las empresas born global. De las empresas ubicadas en la ciudad de Bogotá -121 empresas- y Medellín -81 empresas-. el $73,39 \%$ han aumentado sus exportaciones en más del $20 \%$ en los últimos 3 años y el $83,25 \%$ consideran que la calificación del desempeño de las exportaciones es superior a 8 puntos sobre 10 .

Por último, para el tercer grupo de análisis (intensidad competitiva y sus respectivos resultados exportadores), se percibe lo siguiente:

En la gráfica 5 se observa que un alto porcentaje de las empresas encuestadas (81\%), y que obtuvieron una calificación alta en el desempeño de sus exportaciones, están de acuerdo en que el tamaño del mercado es grande, lo que permite inferir que las empresas perciben una actividad competitiva muy agresiva en el mercado, y que debido al

El tamaño del mercado es grande vs. calificación del desempeño de las exportaciones

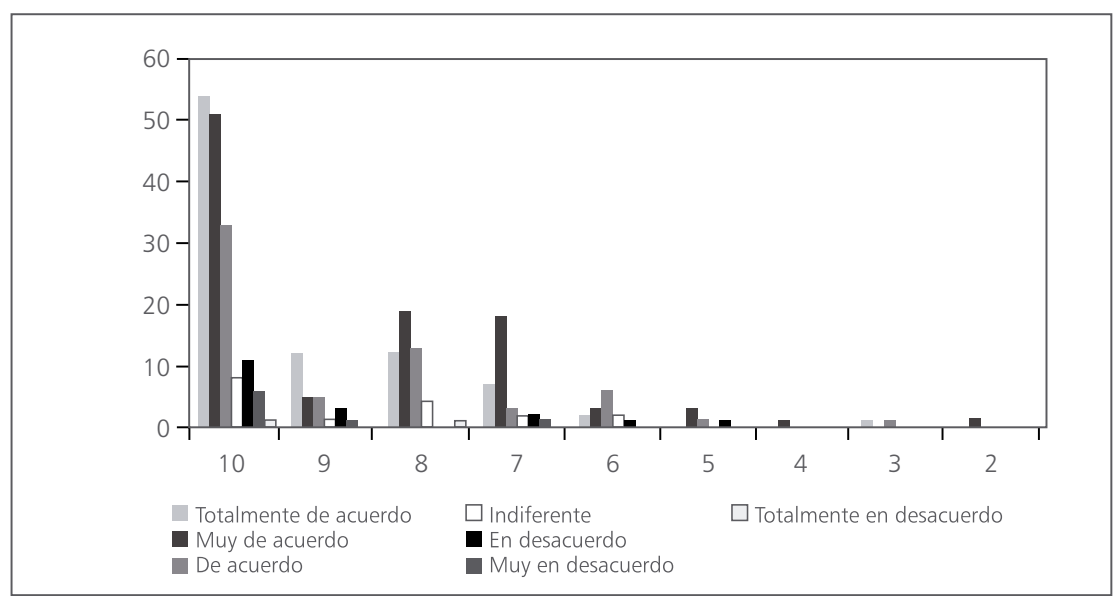

Fuente: Elaboración de los autores. 
resultado exportador favorable, existen muchos competidores que entregan productos o servicios con características similares o poco diferenciados.

Todos estos factores exponen a las empresas born global a elevados niveles de incertidumbre relacionada con los clientes potenciales, sus necesidades y demandas (Hannan \& Freeman, 1984), pero que, a su vez, permite la especialización, la adaptación al cliente y la producción en nicho en los mercados actuales favoreciendo su rápida internacionalización (Madsen \& Servais, 1997). Sin embargo, son los cambios en el mercado los que determinan la aparición de las born global y conllevan al incremento de la especialización y de los nichos de mercado. Esto ocasiona la existencia de empresas que producen componentes o acciones de un producto o servicio, que deben ser vendidos en los mercados internacionales, debido a la demanda insuficiente en los mercados domésticos. Las nuevas condiciones de mercado llevan a las empresas a establecerse en diferentes mercados rápidamente (Madsen \& Servais, 1997).

\section{Análisis de conglomerados}

Tomando como base las variables anteriormente relacionadas y por medio del Programa IBM SPSS Statictics versión 19 , se realiza un análisis de conglomerado bietápico que arroja la siguiente visualización:

Gráfica 6.

Resultado de conglomerado en dos fases

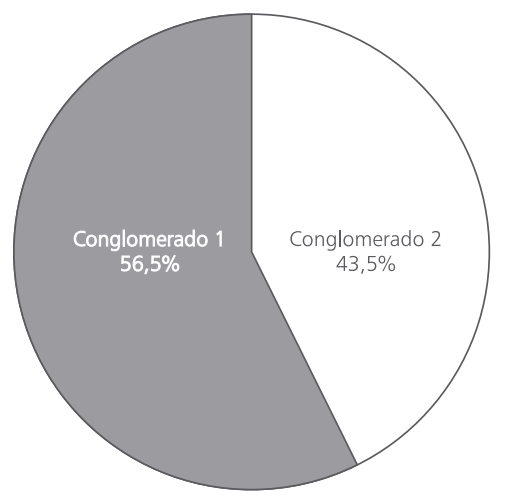

Fuente: Elaboración de los autores.

Resultado conglomerado dos fases

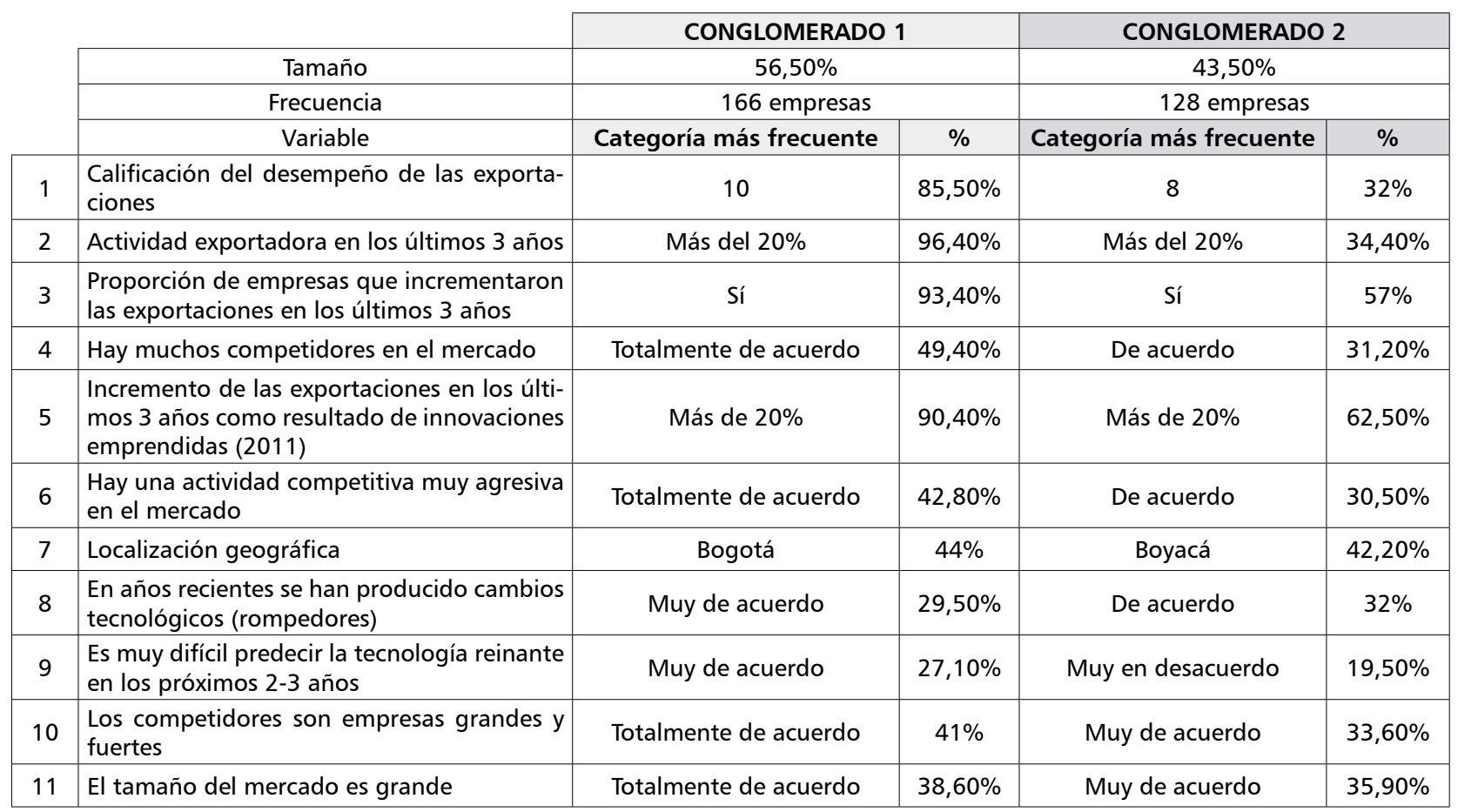

Fuente: Elaboración de los auores. 
El resultado de la gráfica 6 y la tabla 1 permite visualizar que se forman dos conglomerados que agrupan 168 empresas en el primero y 129 empresas en el segundo. A continuación se realizan dos tipos de análisis a los conglomerados resultantes para evidenciar lo común entre ellos (análisis vertical) y las diferencias entre los mismos (análisis horizontal), desde el punto de vista de las variables.

\section{Análisis vertical}

El primer conglomerado o conglomerado 1 aglutina 168 empresas que comparten características similares como el estar de acuerdo en que existen muchos competidores en el mercado y constantes cambios tecnológicos que se pueden considerar rompedores, los cuales hacen difícil predecir la tecnología reinante en los próximos 2-3 años. También consideran que los competidores son empresas grandes y fuertes, que el tamaño del mercado en el que participan es grande y además que este presenta una actividad competitiva muy agresiva. Todo esto les ha permitido aumentar sus exportaciones en los últimos 3 años en más del 16\% y calificar el desempeño de sus exportaciones como exitosas, como resultado de las innovaciones emprendidas en sus empresas. Las principales ubicaciones geográficas son las ciudades de Bogotá y Medellín. La producción y comercialización de los productos y servicios es bastante dinámica. En esto tiene mucha incidencia el rápido y constante avance que tiene la tecnología, lo que ha permitido que los servicios lleguen casi instantáneamente a los consumidores.

El segundo conglomerado se denominará conglomerado 2 y se conforma de 129 empresas. Este conglomerado es más disperso en el resultado de sus variables, debido a que no posee una buena calificación de sus exportaciones y no han aumentado sus exportaciones en los últimos 3 años. Como dato adicional el $42,4 \%$ de estas empresas se encuentra ubicada en Boyacá diferenciándose en gran medida de las ubicaciones predominantes del primer conglomerado. Respecto a los cambios tecnológicos rompedores, las empresas de este conglomerado consideran que este aspecto no es muy representativo de su mercado, esto permite inferir que no realizan actividades tecnológicas importantes que le permitan crear una ventaja competitiva con respecto a sus competidores. Por lo tanto, en este conglomerado no se evidencian variables claras o predominantes que permitan identificar cuáles son importantes o definitivas para estas empresas.

\section{Análisis horizontal}

Este análisis consiste en la valoración del desempeño de las exportaciones en el conglomerado 1 y en el conglomerado 2. Como se puede observar, en general, la mayoría de las empresas de los conglomerados 1 y 2 valoraron el desempeño exportador en un rango de 8 a 10; sin embargo, el resultado del conglomerado 1 tiene una clara tendencia a valorar el desempeño exportador de una manera más homogénea, con el $85 \%$ de las empresas y una calificación de 10. Por otra parte, el resultado para el conglomerado dos es más disperso y, por tanto, la calificación más recurrente es de 8 , esta solo representa al $32 \%$ de las empresas. Adicionalmente, se registran exportaciones no exitosas solo para las empresas integrantes del conglomerado 2 (ver anexo 4).

Ahora bien, en cuanto al porcentaje de la actividad exportadora en los últimos 3 años para los conglomerados 1 y 2 se encontraron los siguientes resultados. En general, la mayoría de las empresas pertenecientes al conglomerado 1 incrementaron la actividad exportadora en más del $20 \%$. De las 166 empresas que conforman el conglomerado 1, el $96,4 \%$ incrementaron las exportaciones en más del $20 \%$; sin embargo, en el caso del segundo conglomerado, de las 128 empresas que lo integran solo el $44 \%$ incrementaron las exportaciones más del $20 \%$. Para el caso del conglomerado 2, la actividad exportadora fue más dispersa, las empresas que lo conforman incrementaron las exportaciones en diversos porcentajes, 31 de estas solo incrementaron del 1 al 5\%, mientras que 6 empresas no exportaron (ver anexo 5). 
Sobre la apreciación de las empresas de los conglomerados 1 y 2, acerca del tamaño del mercado, se encuentra que en el conglomerado 1, el $89 \%$ de las empresas considera que el mercado es grande. En el caso del conglomerado 2, el $80 \%$ de las empresas tienen esta misma apreciación. Adicionalmente, el $11 \%$ de las empresas del conglomerado 2 no están de acuerdo con la afirmación de que el mercado es grande, frente al $8 \%$ del conglomerado 1 (ver anexo 6).

Sobre la apreciación de las empresas de los conglomerados 1 y 2 en relación con la intensidad de la actividad competitiva en el mercado, se encuentra que la intensidad competitiva es homogénea para ambos conglomerados. En general para el conglomerado 1, el 92,2\% de las empresas considera que existe una actividad competitiva muy agresiva en el mercado; en el caso del conglomerado 2, el $89,1 \%$ de las empresas tienen esta misma apreciación. Adicionalmente, el $1,6 \%$ de las empresas del conglomerado 2 consideran que no existe una actividad competitiva muy agresiva frente al 4,2\% del conglomerado 1 (ver anexo 7).

La apreciación de las empresas de los conglomerados 1 y 2 acerca de si en los últimos años se ha producido cambios tecnológicos rompedores presenta dispersión para el conglomerado 2 y más homogeneidad para el conglomerado 1. En general para el conglomerado 1 , el $76,5 \%$ de las empresas considera que han ocurrido cambios tecnológicos rompedores; en el caso del conglomerado 2 solo el $60,2 \%$ de las empresas tienen esta misma apreciación (ver anexo 8).

Al realizar este análisis se identifican las siguientes diferencias que son descritas para cada conglomerado:

El primer conglomerado agrupa empresas ubicadas principalmente en Bogotá. Estas empresas tienen la percepción de que el mercado es grande y que existe una competitividad muy agresiva en él. Por lo tanto son empresas que reconocen la importancia de la tecnología y el impacto de los cambios tecnológicos en sus procesos productivos. Es así como estas empresas poseen procesos de producción y comercialización de productos y servicios más dinámicos. En el mismo sentido, como consecuencia de lo anteriormente mencionado, son compañías que describen un incremento de las exportaciones como resultado de las innovaciones emprendidas.

El segundo conglomerado agrupa empresas ubicadas principalmente en Boyacá. Estas empresas tienen la percepción de que el mercado es grande y que existe una actividad competitiva agresiva en el mercado; sin embargo, no reconocen la importancia de la tecnología y el impacto de esta en sus procesos. Lo anterior se debe en parte a que en estas empresas en los últimos 2-3 años no han ocurrido cambios tecnológicos rompedores y, por lo tanto, la mayoría de estas empresas no realiza innovaciones en busca de incrementar sus exportaciones.

Los conglomerados 1 y 2 se diferencian principalmente por: a) la ubicación geográfica, b) la percepción de la importancia de la tecnología y su impacto, c) los resultados exportadores. Por lo tanto, se puede inferir que las características tecnológicas de las empresas born global en Colombia permiten incrementar en mayor medida el dinamismo exportador (resultados conglomerado 1) y que la ubicación geográfica influye fuertemente en las percepciones de las características del mercado y la tecnología. Todo esto, como lo demuestran los resultados, hace que el conglomerado 1 tenga un mayor dinamismo exportador que el conglomerado 2 .

\section{DISCUSIÓN DE RESULTADOS}

Los resultados del presente artículo conducen a determinar que las empresas born global con mayor dinamismo exportador se caracterizan por pertenecer a un entorno altamente competitivo, compuesto por muchas empresas fuertes, y donde el cambio tecnológico es constante. Se habla de entonces de empresas que se desarrollan en sectores altamente representativos y ubicadas en Bogotá, que, por su condición de capital del país, representa una zona donde las empresas encuentran mayores oportunidades para expandirse. Los tres componentes principales de la dinámica exportadora de 
las empresas born global caracterizados en este estudio, nivel tecnológico, localización geográfica e intensidad competitiva, muestran unos resultados que coinciden con Knigth y Cavusgil (1996) que resaltan la importancia del nivel tecnológico en el comportamiento exportador de las born global. Así como con Madsen, Rasmussen y Servais (2000) que resaltan el alcance geográfico de las empresas, y con Monreal (2009) que considera el comportamiento exportador de las empresas born global basado en los cambios tecnológicos y la rivalidad competitiva.

A nivel de la teoría sobre emprendimiento internacional, las características de la dinámica exportadora identificadas en este artículo contribuyen a la identificación de las oportunidades de negocio que favorecen la creación de nuevas empresas y su internacionalización. Igualmente, además de la conducta innovadora y proactiva que está detrás del concepto inicial de emprendimiento internacional (McDougall \& Oviatt, 2000), también se puede hablar de condiciones de amplitud y dinamismo de los mercados. Adicionalmente, los resultados que se obtienen en Colombia contribuyen a rebatir la creencia de que las empresas nuevas, que además son pymes, poseen unas limitaciones en su capacidad tecnológica y acceso a la información que dificultan su crecimiento económico y sus posibilidades de exportar (Madsen \& Servais, 1997), dado que se prueba que las empresas born global colombianas se arriesgan en mercados altamente competitivos y asumen los cambios tecnológicos de su entorno.

\section{CONCLUSIONES}

Ante el aumento constante de empresas que optan por exportar durante su primeros años de existencia, la internacionalización de nuevas empresas es una temática que logra adquirir en el campo académico una gran acogida y el planteamiento de diferentes inquietudes, (McDougall, Shane \& Oviatt, 1994). Por tanto, con el fin de contribuir en el conocimiento de este tipo de empresas, este trabajo analiza los factores que influyen en el dinamismo del mercado de las empresas tipo born global en Colombia
Se ha pretendido estudiar los efectos de los niveles tecnológicos de las empresas, la influencia de las características sectoriales y la relación de la intensidad competitiva en los resultados exportadores de las empresas born global en Colombia. Asimismo, no existe evidencia de la existencia de trabajos en Colombia que analicen el entorno de forma detallada, aunque pocos reconozcan que es uno de los factores más relevantes en la creación de empresas Born Global (Escandón, 2009).

Dentro de los principales hallazgos se evidencia que las 296 empresas born global de la muestra se agrupan en sectores clasificados como intensivos en tecnología e innovación, tales como: fabricación de sustancias y productos químicos (54 empresas); fabricación de productos de caucho y de plástico (51 empresas); elaboración de productos alimenticios y bebidas (44 empresas), y fabricación de productos textiles (28 empresas), estas representan más del $61 \%$ del total de las empresas born global.

Las principales ciudades en Colombia que se han posicionado en el escenario mundial de un modo atractivo e integrado a una red global, con un buen desarrollo de las TIC y con mayores facilidades de trámites, son: Bogotá con el $41 \%$; $27 \%$, en Medellín; $21 \%$, en Boyacá; $10 \%$, en Cali, $y$ el $1 \%$, en Pereira.

Adicionalmente se resalta dentro de este estudio que el $73 \%$ de las empresas que se encuentran ubicadas en las ciudades de Bogotá y Medellín han aumentado sus exportaciones en más del $20 \%$ en los últimos 3 años y el $83 \%$ de estas consideran que la calificación del desempeño de las exportaciones es superior a 8 puntos sobre 10. Por lo tanto, la influencia de la ubicación geográfica está directamente relacionada con el resultado de las exportaciones en las empresas born global.

Dada la tendencia de las empresas born global a reconocer que existen muchos competidores en el mercado y que los competidores son empresas grandes y fuertes, los empresarios son conscientes de que la única forma de mantenerse en el mercado internacional es por medio del me- 
joramiento de sus procesos o productos como una forma de mejorar sus niveles de especialización y su adaptación a las necesidades del cliente.

Se evidencia la importancia de la intensidad competitiva en los sectores y los efectos en los resultados exportadores, asociado a la tendencia de que las empresas born global se comportan de una manera muy activa en los mercados internacionales desde una edad temprana. Esto se ve reflejado en que el $74 \%$ inician la exportación de sus productos antes de cuatro años y el $69 \%$ incrementan sus exportaciones en más del $20 \%$ anualmente.

Por último, se encuentra que las empresas born global en Colombia perciben el mercado como grande, con una actividad competitiva muy agresiva debido a los elevados niveles de incertidumbre a los que están expuestas. Estos niveles son dados por la necesidades y demandas de sus clientes potenciales, que en últimas las llevan a buscar la especialización, la adaptación y la producción de productos específicos que sean aceptados en lugares donde el mercado doméstico es insuficiente o incapaz. Es por esto que al encontrar una condición de mercado insatisfecho, estas se posicionan rápidamente incrementando su dinamismo exportador generalmente a una edad temprana.

Con respecto a estos resultados, se pueden obtener importantes consecuencias para mejorar el desempeño de este tipo de empresas en Colombia y para guiar a los nuevos empresarios en sus procesos de creación de empresas. Los empresarios y las instituciones encargadas de implementar la política pública deben buscar la forma de promover la adopción de tecnologías avanzadas, con el fin de hacer más competitivas las empresas. De igual forma, debido a la importancia de las empresas born global que logran desarrollar capacidades organizacionales que les permiten competir en mercados grandes y competitivos, es relevante pensar en hacer un mayor énfasis en la creación de empresas con potencial exportador y formalizar organizaciones de seguimiento y apoyo a su gestión, dada su relevancia en el desarrollo económico de la región.
A partir del análisis realizado en este artículo, es posible que las empresas, personas, y el Gobierno puedan entender la importancia de facilitar el proceso de internacionalización temprana a las empresas, así como la necesidad de generar condiciones para la creación de un ventaja competitiva en las organizaciones en pro de su fortalecimiento para enfrentarse a mercados competitivos y el aprovechamiento de las oportunidades de expansión que los tratados de libre comercio pueden brindan para el crecimiento económico.

Las organizaciones, por su parte, deben comprender que la ventaja competitiva para enfrentarse a nuevos mercados internacionales no solo se adquiere estableciendo redes empresariales, sino que estas deben ir acompañadas de la capacidad de enfrentar los cambios constantes en el mercado, para crear valor agregado para el cliente final y el lograr una ventaja frente a los competidores.

Dentro de las limitantes de este estudio, se encuentra, en primer lugar, que los resultados obtenidos caracterizan el entorno empresarial colombiano y, a pesar de ser consecuentes con la evidencia empírica sobre el tema, no es prudente generalizar estos resultados para otros países. De igual forma, aunque la metodología utilizada permite identificar las características que identifican la dinámica exportadora de las empresas born global, especialmente las características del entorno exportador de una empresa exitosa y no exitosa, podrían aplicarse otro tipo de metodologías que brinden indicadores individuales de cada una de estas variables e incluso incluir nuevas variables que, por efectos del contexto teórico expuesto, quedaron por fuera del estudio. Como líneas futuras de investigación, sería interesante hacer comparativos entre los factores externos que afectan la dinámica exportadora, frente a los factores internos organizacionales, con el fin de generar un marco global del comportamiento exportador de las born global en Colombia. Además, teniendo en cuenta que ya se tienen los primeros resultados de los tratados de libre comercio firmados recientemente, resulta importante incorporar, como variables de estudio, las ventajas que ofrecen estos tratados. 


\section{REFERENCIAS}

1. Aaby, N. E. \& Slater, S. F. (1989). Management Influences on Export Performance: A Review of the Empirical Literature 1978-88. International Marketing Review, 6(4), 7-26.

2. Acedo, F. J. \& Jones, M. V. (2007). Speed of Internationalization and Entrepreneurial Cognition: Insights and a Comparison between International New Ventures, Exporters and Domestic Firms. Journal of World Business, 42, 236-252.

3. Andersen, O. \& Buvik, A. (2002). Firms' Internationalization and Alternative Approaches to the International Customer/Market Selection. International Business Review, 11, 347-363.

4. ANIF. (2012). La gran encuesta Pyme. Segundo semestre 2012 - Informe de resultados. Recuperado de http://anif.co/sites/default/files/uploads/GranEncuesta\%20I-2012.pdf.

5. Autio, E., Sapienza, H. \& Almeida, J. (2000). Effects of Age at Entry, Knowledge Intensity, and Imitability on International Growth. Academy of Management Journal, 43, 909-924.

6. Autio, E. \& Sapienza, H. (2000). Comparing Process and Born Global Perspectives in them International Growth of Technology-Based New Firms. Frontiers of Entrepreneurship Research (pp. 413-424). Wellesley, Massachusetts: Center for Entrepreneurial Studies, Babson College.

7. Bain, J. (1959). Industrial Organization. New York, NY: Wiley.

8. Bartlett, C. \& Ghoshal, S. (1991). Managing Across Borders: The Transnational Solution. Boston: Harvard Business School Press.

9. Bell, J. (1995). The Internationalization of Small Computer Software Firms: A Further Challenge to Stage Theories. European Journal of Marketing, 29(8), 60-75.

10. Bloodgood, J., Sapienza, H. \& Almeida, J. (1996). The internationalization of new high-potential U.S. ventures: antecedents and outcomes. Entrepreneurship: Theory and Practice, 20(4), 61-76.

11. Blomqvist, K., Hurmelinna-Laukkanen, P., Nummela, N. \& Saarenketo, S. (2008). The Role of Trust and Contracts in the Internationalization of Technology-Intensive Born Globals. Journal of Engineering and Technology Management, 25(1/2), 123-135.

12. Carpano, C., Chrisman, J. \& Roth, K. (1994). International Strategy and Environment: An Assessment of the Performance Relationship. Journal of International Business Studies, 25, 639-656.

13. Confecámaras. (2012). Oportunidades y retos de las regiones de Colombia frente a los tratados de libre comercio. Recuperado de http://www.confecamaras.org.co/phocadownload/Libros/ Cuaderno\%20de\%20An\%C3\%A1lisis\%20Econ\%C3\%B3mico\%20No.\%202.pdf.

14. Cuervo, A., Ribeiro, D. \& Roig, S. (2007). Entrepreneurship: Conceptos, teoría y perspectiva. Introducción. En A. Cuervo, D. Ribeiro \& S. Roig (Eds.), Entrepreneurship: Conceptos, teoría y perspectiva (pp. 9-25). Valencia: Fundación Bancaja.

15. Díaz, B., Moniche, L. \& Morillas, M. (2005). Identificación de sectores clave mediante análisis de conglomerados difuso. El caso de España. I Jornada de Análisis Input-Output. 22 y 23 de septiembre. Oviedo, España.

16. Dimitratos, P. \& Plakoyiannaki, E. (2003). Theoretical Foundations of an International Entrepreneurial Culture. Journal of International Entrepreneurship, 1, 187-215.

17. Dimitratos, P. \& Jones, M.V. (2005). International Entrepreneurial Culture, International Opportunity Perception and Pattern of International Exploitation: Towards an Integrated Model. In P. Ghauri, A. Hadhikani \& J. Johansonn (Eds.), Managing Opportunity Development in Business Networks (pp. 67-90). Houndmills, UK: Palgrave. 
18. Dunning, J. H. (1958). American Investment in British Manufacturing Industry. London: Allen \& Unwin. Reprinted by Arno Press, New York.

19. Engwall, L. \& Wallenstal, M. (1988). Tit for Tat in Small Steps: The Internationalization of Swedish Banks. Scandinavian Journal of Management, 4(3/4), 147-155.

20. Escandón, D. (2009). Factores que inciden en la creación de born global en Colombia. Estudios Gerenciales, 25(113), 55-73.

21. Escandón, D. \& Hurtado, A. (2012). Características de los directivos de las empresas Born Global en Colombia. Entramado, 8(2), 74-93.

22. Fernández, M., Peña-García, I. \& Hernández, F. (2008). Factores determinantes del éxito exportador. El papel de la estrategia exportadora en las cooperativas agrarias. Revista de Economía Pública, social y cooperativa. 63, 39-64.

23. Flor, M. \& Oltra, M. (2009). La estrategia exportadora de la empresa y su relación con el resultado internacional. Investigaciones europeas de dirección de la empresa (IEDEE), 16(Iss. 1), 15-29.

24. Galván, I. (2003). La formación de la estrategia de selección de mercados exteriores en el proceso de internacionalización de las empresas (Tesis doctoral). Universidad de Palmas de Gran Canaria, España.

25. González, J., Peña, I. \& Vendrell, F. (2012). Innovation, Entrepreneurial Activity and Competitiveness at a Sub-National Level. Small Business Economics, 39(3), 561-574.

26. Hair, J., Anderson, R., Tatham, R. \& Black, W. (1999). Análisis multivariante (5ª edición). Madrid: ed. Prentice Hall Iberia.

27. Hannan, M. \& Freeman, J. (1984). Structural Inertia and Organizational Change. American Sociological Review, 49, 149-164.

28. Hymer, S. H. (1960) The International Operations of National Firms: A Study of Direct Investment (Tesis doctoral). MIT, Boston.

29. Iborra, M., Menguzzato, M. \& Ripollés, M. (1998). Creación de empresas internacionales: Redes informales y obtención de recursos. Revista Europea de Dirección y Economía de la Empresa, $7(3), 147-160$.

30. Jap, S. (1999). Pie-Expansion Efforts: Collaboration Processes in Buyer-Seller Relationships. Journal of Marketing Research, 36(4), 461-475.

31. Jaworski, B. \& Kohli, A. (1993). Market Orientation: Antecedents and Consequences. The Journal of Marketing, 57(3), 53-70.

32. Johanson, J. \& Vahlne, J. (1977). The Internationalization Process of the Firm: A Model of Knowledge Development and Increasing Foreign Market Commitment. Journal of International Business Studies, 8(1), 23-32.

33. Jolly, V., Alahunta, M. \& Jeannet, J. (1992). Challenging the Incumbents: How High Technology Start-Ups Compete Globally. Journal of Strategic Change, 1, 71-82.

34. Jones, M., Coviello, N. \& Tang, Y. (2011). International Entrepreneurship Research (1989. 2009): A Domain Ontology and Thematic Analysis. Journal of Business Venturing, 26, 632-659.

35. Katsikeas, C. S., Piercy, N. F. \& Ioannidis, C. (1996) Determinants of Export Performance in a European Context. European Journal of Marketing, 30(6), 6-35.

36. Kihlstrom, R. \& Laffont, J. (1979). General Equilibrium Entrepreneurial Theory of Firm Formation Based on Risk Aversion. Journal of Political Economy, 87, 719-748. 
37. Knight, G. (2000). Entrepreneurship and Marketing Strategy: The SME under Globalization. Journal of International Marketing, 8(2), 12-32.

38. Knight, G. A. \& Cavusgil, T. (1996). The Born Global Firm: A Challenge to Traditional Internationalization Theory. Advances in International Marketing, 8, 11-26.

39. Knight, G. A. \& Cavusgil, T. (2004). Innovation, Organizational Capabilities, and the BornGlobal Firm. Journal of International Business Studies, 35, 124-141.

40. Knight, G. A., Madsen, T. \& Servais, P. (2004). An Inquiry into Born-global Firms in Europe and the USA. International Marketing Review, 21 (6), 645-665.

41. Kohli, A. \& Jaworski, B. (1990). Market Orientation: The Construct, Research Propositions, and Managerial Implications. Journal of Marketing, 54, 1-18.

42. Kohli, A., Jaworski, B. \& Kumar, A. (1993). MARKOR: A Measure of Market Orientation. Journal of Marketing Research, XXX, 467-477.

43. Kuivalainen, O., Sundqvist, S. \& Servais, P. (2007). Firms' Degree of Born-Globalness, International Entrepreneurial Orientation and Export Performance. Journal of World Business, 42, 253-267.

44. Lumpkin, G. \& Dess, G. (1996). Clarifying the Entrepreneurial Orientation Construct and Linking it to Performance. Academy of Management Review, 21 (1), 135-172.

45. Luo, Y. \& Park, S. H. (2001). Strategic Alignment and Performance of Market-Seeking MNCs in China. Strategic Management Journal, 22(2), 141-155.

46. Luostarinen, R. \& Gabrielsson, M. (2004). Finnish Perspectives of International Entrepreneurship. In L. P. Dana (Ed.), Handbook of Research on International Entrepreneurship (pp. 383-403). Cheltenham: Edward Elgar.

47. Madsen, T. K. (1987). Empirical Export Performance Studies: A Review of Conceptualizations and Findings. Advances in International Marketing, 2, 177-198.

48. Madsen, T. \& Servais, P. (1997). The Internationalization of Born Global: An Evolutionary Process? International Business Review, 6(6), 561-583.

49. Madsen, T., Rasmussen, E. \& Servais, P. (2000). Differences and Similarities between Born Globals and other Types of Exporters. Advances in International Marketing, 10, 247-265.

50. McAuley, A. (1999). Entrepreneurial Instant Exporters in the Scottish Arts and Crafts Sector. Journal of International Marketing, 7(4), 67-82.

51. McDougall, P. \& Oviatt, B. (1996). New Venture Internationalization, Strategic Change, and Performance: A Follow-up Study. Journal of Business Venturing, 11(1), 23-40.

52. McDougall, P. \& Oviatt, B. (2000). International Entrepreneurship: The Intersection of Two Research Paths. Academy of Management Journal, 43, 902-908.

53. McKinsey y Co. (1993). Emerging Exporters: Australia's High Value-Added Manufacturing Exporters. Melbourne: Australian Manufacturing Council.

54. Milanov, H. \& Fernhaber, S. A. (2009). The Impact of Early Imprinting on the Evolution of New Venture Networks. Journal of Business Venturing, 24(1), 46-61.

55. Ministerio de Comercio, Industria y Turismo. (2012). Colombia avanza en comercio exterior, atracción de IED, turismo y apoyo a empresarios. Recuperado de https://www.mincomercio.gov. co/publicaciones.php?id=4528. 
56. Mitchell, J. (1969). The Concept and Use of Social Networks. En J. Mitchell (Ed.), Social Networks in Urban Situations: Analyses of Personal Relationships in Central African Town (pp. 1-50). Manchester: Manchester University Press.

57. Moen, O. (1999). The Relationship between Firm Size, Competitive Advantages and Export Performance Revisited. International Small Business Journal, 18(1), 53-71.

58. Moen, O. (2002). The Born Globals: A New Generation of Small European Exporters. International Marketing Review, 19(Iss: 2), 156-175.

59. Monreal, J. (2009). Análisis del comportamiento exportador de la empresa española desde el enfoque de los recursos y capacidades (Tesis doctoral). Universidad de Murcia, España.

60. Nordman, E. \& Melen, S. (2008). The Impact of Different Kinds of Knowledge for the Internationalization Process of Born Globals in yhe Biotech Business. Journal of World Business, $43(2), 171-185$.

61. Ohe, T., Honjo, S., Oliva, M. \& MacMillan, I.C. (1991). Entrepreneurs in Japan and Silicon Valley: A Study of Perceived Differences. Journal of Business Venturing, 6(2), 135-144.

62. Oviatt, B. \& McDougall, P. (1994). Toward a Theory of International New Ventures. Journal of International Business Studies, 25(1), 45-64.

63. Oviatt, B. \& McDougall, P. (1995). Global Start-Ups: Entrepreneurs on a Wold-Wide Stage. Academy of Management Executive, 9(2), 30-43.

64. Oviatt, B. \& McDougall, P. (2005). Defining International Entrepreneurship and Modeling the Speed of Internationalization. Entrepreneurship: Theory and Practice, 29(5), 537-553.

65. Perks, K. \& Hughes, M. (2008). Entrepreneurial Decision-Making in Internationalization: Propositions from Mid-Size Firms. International Business Review, 17(3), 310-330.

66. Rasmussan, E. S., Madsen, T. K. \& Evangelista, F. (2001). The Founding of the Born Global Company in Denmark and Australia: Sensemakin and Networking. Asia Pacific Journal of Marketing and Logistics, 13(3), 75-107.

67. Rennie, M. (1993). Global Competitiveness: Born Global. McKinsey Quarterly, 4, 45-52.

68. Rialp, A. (1999). Los enfoques microorganizativos de la internacionalización de la empresa: una revisión y síntesis de la literatura. Información comercial española. Revista de Economía, $781,117-128$.

69. Rialp, A. \& Rialp, J. (2001). Conceptual Framewoks on Smes Internationalization: Past, Present, and Future Trends of Research. In C. N. Axinn \& P. Matthyssens (Eds.), Reassessing the Internationalization of the Firm (Advances in International Marketing 11, pp. 49-78). Amsterdam: JAI/Elsevier.

70. Rialp, A., Rialp, J. \& Salas, V. (2002). Faster and Highly Successful Exporters: An Explanation of Born Globals Firms from the Resource-Based View. Proceedings of RENT XVI (pp. 355-387). Barcelona.

71. Rialp, A., Rialp, J. \& Knight, G. (2005). The Phenomenon of Early Internationalizing Firms: What do We Know after a Decade (1993-2003) of Scientific Inquiry? International Business Review, 14(2), 147-166.

72. Ripollés, M., Menguzzato, M. \& Iborra, M. (2002). The Internationalisation of New Ventures. The Spanish Case. International Journal of Entrepreneurship and Innovation, 3(3), 191-200. 
73. Rossman, M. (1984). Export Trading Company Legislation: U.S. Response to Japanese Foreign Market Penetration. Journal of Small Business Management, 22 (4), 62-66.

74. Schwarz, G. (1978). Estimating the Dimension of a Model. The Annals of Statistics, 6(2), 461-464.

75. Servais, P., Zucchella, A. \& Palamara, G. (2006). International Entrepreneurship and Sourcing: International Value Chain of Small Firms. Journal of Euromarketing, 16(1), 105-117.

76. Shane, S. \& Venkataraman, S. (2000). The Promise of Entrepreneurship as a Field of Research. Academy of Management Review, 25, 217-226.

77. Shoham, A. (1999). Bounded Rationality, Planning, Standardization of International Strategy, and Export Performance: A Structural Model Examination. Strategic Management Journal, $9(1), 43-60$.

78. Shrader, R., Oviatt, B. \& McDougall (2000). How New Venture Exploit Trade-Offs Among International Risk Factors: Lessons for the Accelerated Internationalization of the $21 \mathrm{St}$ Century. Academy of Management Journal, 43(6), 1127-1247.

79. Sousa, C., Martínez-López, F. \& Coelho, F. (2008). The Determinants of Export Performance: A Review of the Research in the Literature between 1998 and 2005. International Journal of Management Reviews, 10(4), 343-374.

80. Tichy, N. (1981). Networks in Organization. En P. Nystrom \& W. Starbuck (Eds.), Handbook of Organizational Design (pp. 225-249). New York, NY: Oxford University Press.

81. Tushman, M. \& Anderson, P. (1986). Technological Discontinuties and Organizational Environments. Administrative Science Quarterly, 31, 439-465.

82. Weimann, G. (1989). Social Networks and Communication. En M. Asante \& W. Gudykunst (Eds.), Handbook of International and Intercultural Communication. Newbury Park, CA: Sage.

83. Young, S. (1987). Business Strategy and the Internalization of Business: Recent Approaches. Managerial and Decisions Economics, 8, 31-40.

84. Zahra, S. A. (2005). Entrepreneurial Risk Taking in Family Firms. Family Business Review, $18,23-40$.

85. Zahra, S. \& George, G. (2002). Absorptive Capacity: A Review, Reconceptualization, and Extension. Academy of Management Review, 27(2), 185-203.

86. Zahra, S., Ireland, R. \& Hitt, M. A. (2000). International Expansion by New Venture Firms: International Diversity, Mode of Market Entry, Technological Learning and Performance. Academy of Management Journal, 43, 925-950.

87. Zou, S., Taylor, Ch. \& Osland, G. (1998). The EXPERF Scale: A Crossnational Generalized Export Performance Measure. Journal of International Marketing, 6(3), 10.

88. Zucchella, A., Palamara, G. \& Denicolai, S. (2007). The Drivers of the Early Internationalization of the Firm. Journal of World Business, 42, 268-270. 


\section{ANEXOS}

\section{Anexo 1}

\begin{tabular}{|c|c|}
\hline CIIU & CIIU DESCRIPCION \\
\hline 1 & Agricultura, Ganadería, caza y actividades de servicios conexas \\
\hline 15 & Elaboración de productos alimenticios y de bebidas \\
\hline 17 & Fabricación de productos textiles \\
\hline 18 & Fabricación de prendas de vestir; preparado y tejido de pieles \\
\hline 19 & Curtido y preparado de cueros; fabricación de calzado; fabricación de artículos de viaje, maletas. \\
\hline 2 & Silvicultura, extracción de madera y actividades de servicios conexas \\
\hline 20 & Transformación de la madera y fabricación de productos de madera y de corcho, excepto muebles; fabricación \\
\hline 21 & Fabricación de papel, cartón y productos de papel y cartón \\
\hline 22 & Actividades de edición e impresión y de reproducción de grabaciones \\
\hline 23 & Coquización, fabricación de productos de la refinación del petróleo y combustible nuclear \\
\hline 24 & Fabricación de sustancias y productos químicos \\
\hline 25 & Fabricación de productos de caucho y de plástico \\
\hline 26 & Fabricación de otros productos minerales no metálicos \\
\hline 27 & Fabricación de productos metalúrgicos básicos \\
\hline 28 & Fabricación de productos elaborados de metal, excepto maquinaria y equipo \\
\hline 29 & Fabricación de maquinaria y equipo NCP \\
\hline 3 & No asignada: Acorde con la revisión CIIU_rev_1_ \\
\hline 31 & Fabricación de maquinaria y aparatos el \\
\hline 33 & Fabricación de instrumentos médicos, ópticos y de precisión y fabricación de relojes \\
\hline 34 & Fabricación de vehículos automotores, remolques y semiremolques \\
\hline 36 & Fabricación de muebles; industrias manufactureras NCP \\
\hline
\end{tabular}

Fuente: Banco de la República. Recuperado de http://quimbaya.banrep.gov.co/servicios/saf2/BRCodigosCIIU.html 


\section{Anexo 2}

Código CIIU vs. Proporción de empresas que incrementaron las exportaciones en los últimos 3 años

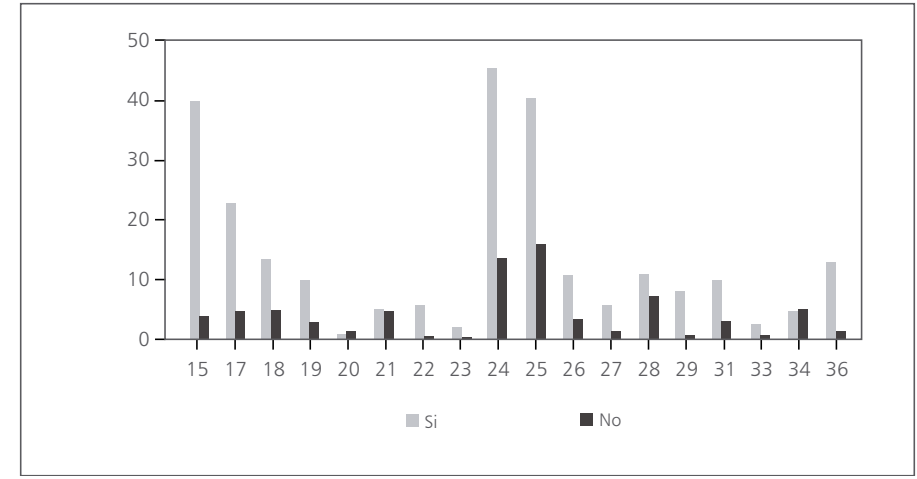

Fuente: Elaboración de los autores.

\section{Anexo 3}

Localización geográfica vs. Actividad exportadora en los últimos 3 años

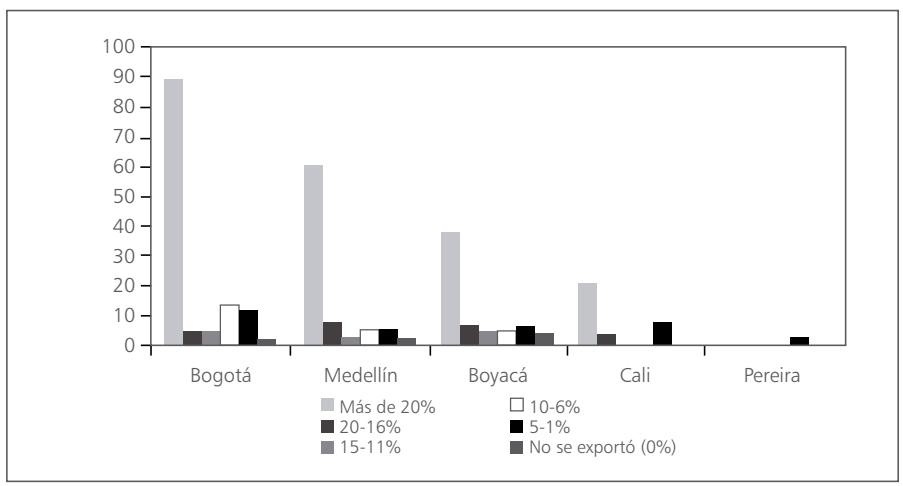

Fuente: Elaboración de los autores.

\section{Anexo 4}

Calificación del desempeño de las exportaciones conglomerado 1 y conglomerado 2

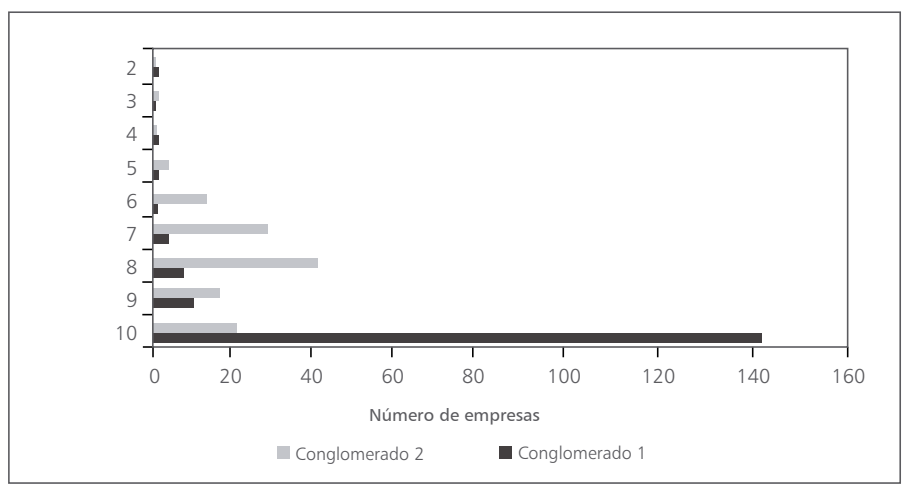

Fuente: Elaboración de los autores. 


\section{Anexo 5}

Actividad exportadora en los últimos 3 años conglomerado 1 y conglomerado 2

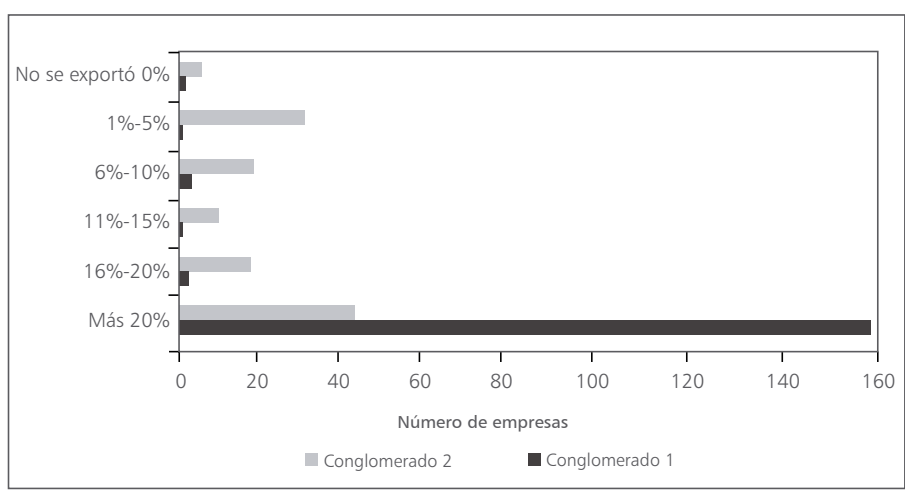

Fuente: Elaboración de los autores.

\section{Anexo 6}

El tamaño del mercado es grande conglomerado 1 y conglomerado 2

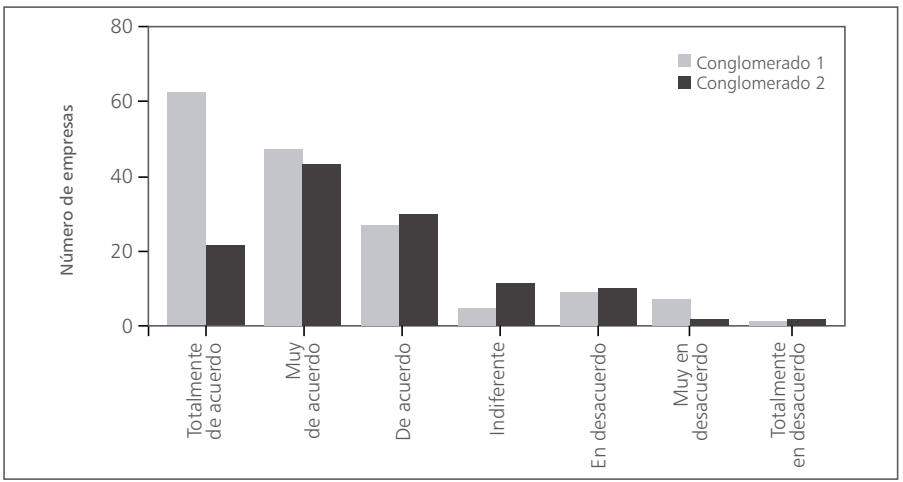

Fuente: Elaboración de los autores.

\section{Anexo 7}

Hay una actividad competitiva muy agresiva en el mercado conglomerado 1 y 2

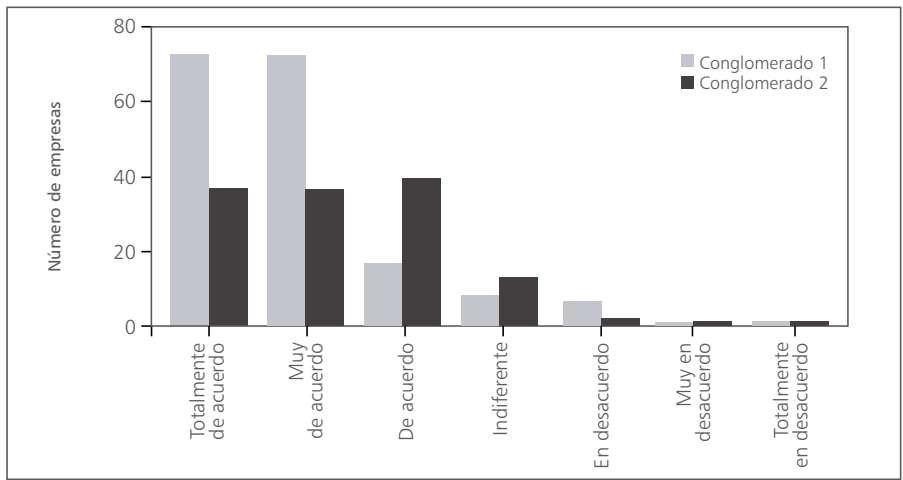

Fuente: Elaboración de los autores. 
Diana Marcela Escandón Barbosa • Andrea Hurtado Ayala • Carlos Alberto Castillo López

\section{Anexo 8}

En años recientes se han producido cambios tecnológicos (rompedores) conglomerado 1 y conglomerado 2.

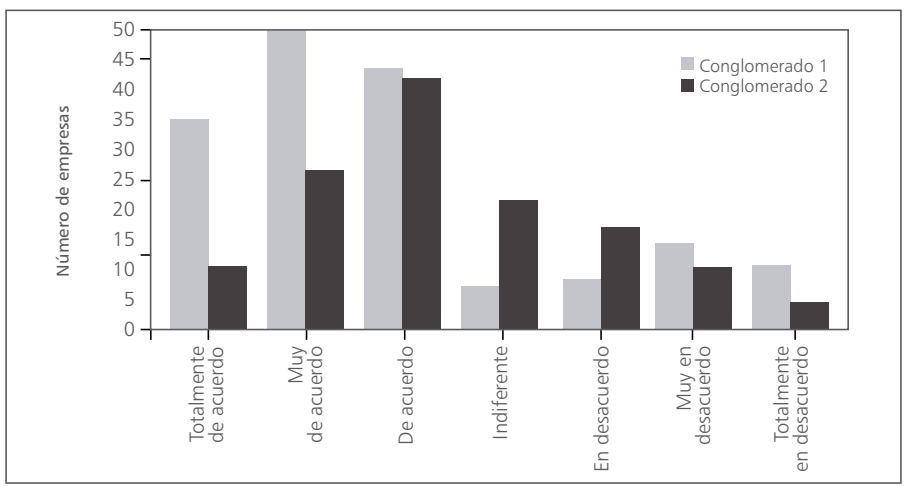

Fuente: Elaboración de los autores. 